\title{
Galaxy cluster X-ray luminosity scaling relations from a representative local sample (REXCESS)
}

\author{
G. W. Pratt ${ }^{1}$, J. H. Croston ${ }^{2}$, M. Arnaud ${ }^{3}$, and H. Böhringer ${ }^{1}$ \\ 1 Max-Planck-Institut für extraterrestriche Physik, Giessenbachstraße, 85748 Garching, Germany \\ e-mail: gwp@mpe.mpg.de \\ 2 Centre for Astrophysics Research, Science and Technology Research Institute, University of Hertfordshire, College Lane, \\ Hatfield AL10 9AB, UK \\ 3 Laboratoire AIM, DAPNIA/Service d'Astrophysique - CEA/DSM - CNRS - Université Paris Diderot, Bât. 709, CEA-Saclay, \\ 91191 Gif-sur-Yvette Cedex, France
}

Received 19 September 2008 / Accepted 20 February 2009

\begin{abstract}
We examine the X-ray luminosity scaling relations of 31 nearby galaxy clusters from the Representative XMM-Newton Cluster Structure Survey (REXCESS). The objects are selected only in X-ray luminosity, optimally sampling the cluster luminosity function. Temperatures range from 2 to $9 \mathrm{keV}$, and there is no bias toward any particular morphological type. To reduce measurement scatter we extract pertinent values in an aperture corresponding to $R_{500}$, estimated using the tight correlation between $Y_{\mathrm{X}}$ (the product of gas mass and temperature) and total mass. The data exhibit power law relations between bolometric X-ray luminosity and temperature, $Y_{\mathrm{X}}$ and total mass, all with slopes that are significantly steeper than self-similar expectations. We examine the possible causes for the steepening, finding that structural variations have little effect and that the primary driver appears to be a systematic variation of the gas content with mass. Scatter about the relations is dominated in all cases by the presence of cool cores. The natural logarithmic scatter about the raw X-ray luminosity-temperature relation is about 70 per cent, and about the X-ray luminosity- $Y_{\mathrm{X}}$ relation it is 40 per cent. Systems with more morphological substructure show similar scatter about scaling relations than clusters with less substructure, due to the preponderance of cool core systems in the regular cluster subsample. Cool core and morphologically disturbed systems occupy distinct regions in the residual space with respect to the best fitting mean relation, the former lying systematically at the high luminosity side, the latter lying systematically at the low luminosity side. Simple exclusion of the central regions serves to reduce the scatter about the scaling relations by more than a factor of two. The scatter reduces by a similar amount with the use of the central gas density as a third parameter. Using $Y_{\mathrm{X}}$ as a total mass proxy, we derive a Malmquist bias-corrected local luminosity-mass relation and compare with other recent determinations. Our results indicate that luminosity can be a reliable mass proxy with controllable scatter, which has important implications for upcoming all-sky cluster surveys, such as those to be undertaken with Planck and eROSITA, and ultimately for the use of the cluster population for cosmological purposes.
\end{abstract}

Key words. X-rays: galaxies: clusters - galaxies: clusters: general - intergalactic medium - cosmology: observations - dark matter

\section{Introduction}

The X-ray luminosity is an observationally attractive quantity because of the relative ease with which it can be measured, and thus it is a key parameter for cosmological applications of the galaxy cluster population. For a fully virialised cluster formed through pure gravitational collapse, the X-ray luminosity $L$ is determined solely by the mass and distribution of gas in the intracluster medium (ICM), and the X-ray temperature $T$ is determined by the depth of the potential well in which the ICM rests. Correlations between these two basic quantities were found in the very early days of X-ray observations of clusters, even while the thermal nature of the emission was still under debate (Mitchell et al. 1977; Mushotzky et al. 1978; Henry \& Tucker 1979). Initial results from these works suggested that the slope of the luminosity temperature relation was steeper than expected from gravitational collapse alone. The launch of the EXOSAT and Einstein observatories enabled the first systematic studies of large samples of clusters (Edge \& Stewart 1991; David et al. 1993), and the subsequent launch of ROSAT, ASCA and Ginga allowed further investigation with increasingly better quality data.
The density squared $\left(n_{e}^{2}\right)$ dependence of the X-ray emission means that luminosity measurements are very sensitive to the exact physics of the gas near the cluster core. Mechanisms such as rapid radiative cooling or merging can change the thermodynamic state of this core gas, introducing scatter into the various luminosity scaling relations. Since our knowledge of the absolute extent of the scatter limits the constraints that can be put on cosmological models with the cluster population, the magnitude of the scatter, its source(s), and how to correct for it constitute some of the most important open issues in the study of clusters (see e.g. Lima \& Hu 2005). Fabian et al. (1994) were the first to note that the offset of a cluster from the mean relation was connected to the presence of a cool core, motivating examination of methods to correct for this effect. Markevitch (1998) derived quantities corrected for the presence of cooling cores by exclusion of the emission from the central region and the introduction of a second spectral component in the temperature estimation; Arnaud \& Evrard (1999) determined the luminosity temperature relation using clusters specifically chosen to have weak or nonexistent cool cores. More recent efforts have aimed at reducing scatter by using the peak surface brightness as a third parameter (O'Hara et al. 2006). At the same time it had long been suspected 
that merging events also contributed to scatter about the mean relation. This effect has been investigated with increasingly sophisticated numerical simulations, which have shown that while major mergers can indeed boost both luminosity and temperature, the boosting appears to be short lived and the net movement in the luminosity temperature plane is approximately parallel to the mean relation (Ricker \& Sarazin 2001; Ritchie \& Thomas 2002; Hartley et al. 2008).

In the present paper we re-investigate the luminosity scaling relations with REXCESS (Böhringer et al. 2007), a sample of 33 local $(z<0.2)$ clusters drawn from the REFLEX catalogue (Böhringer et al. 2004), all of which have been observed with a single satellite, XMM-Newton, with the aim of minimising effects due to instrumental cross calibration uncertainties. The unique sample selection strategy, in which clusters have been selected by luminosity only, in such a way as to have close to homogeneous coverage of luminosity space, delivers an optimal sampling of the luminosity function of the cluster population with no bias towards any particular morphological type. Moreover, distances were optimised so that the angular scale of the objects is such that $\sim R_{500}$ falls well within the XMM-Newton field of view, allowing detailed local background modelling to be undertaken, increasing the precision of measurements at large radii as compared to more nearby clusters which often fill the field of view. Since the basic selection criterion is X-ray luminosity, REXCESS should be representative of any local, unbiased high-quality X-ray survey, of the type applicable to testing of cosmological models.

In the following, we first use the representative nature of the REXCESS sample to investigate the raw luminosity scaling relations, finding that the slopes are steeper than expected if the gas is heated purely by gravitational processes and that cooling cores are the dominant contributor to scatter about them. Dividing the data into subsamples, we investigate the effect of cool cores and morphological disturbance on a cluster's position with respect to the mean relation, finding that the former lie systematically at the high luminosity side, and the latter lie systematically at the low luminosity side. We then investigate two different methods to minimise scatter: simple exclusion of the central region, and use of the central gas density as a third parameter in scaling law fitting, finding that both methods result in a significant reduction in the dispersion about the best fitting relations. Lastly, we examine the physical causes of the steep slope of the luminosity scaling relations, concluding that variations of gas content with total mass are most likely the dominant reason why these are steeper than expected. Our Appendix details the REXCESS survey volume calculations and a first attempt at correcting for Malmquist bias in the luminosity-mass relation.

We adopt a $\Lambda$ CDM cosmology with $H_{0}=70 \mathrm{~km} \mathrm{~s}^{-1} \mathrm{Mpc}^{-1}$, $\Omega_{M}=0.3$ and $\Omega_{\Lambda}=0.7$, and all uncertainties are quoted at the 68 percent confidence level. All logarithmic quantities are given to base $e$, and the quantity $L$ refers to the bolometric [0.01-100 keV] X-ray luminosity.

\section{Data analysis}

Full details of the sample, including XMM-Newton observation details, can be found in Böhringer et al. (2007). Two of the REXCESS clusters, RXC J0956.4-1004 (the Abell 901/902 supercluster) and J2157.4-0747 (a bimodal cluster), display complex morphology and are excluded from the present analysis. The basic characteristics of the clusters discussed in this paper are given in Table 1.

\subsection{Scaling}

In order to estimate cluster quantities consistently, we define them in terms of $R_{500}$, the radius at which the mean mass density is 500 times the critical density at the cluster redshift ${ }^{1}$. While $R_{500}$ can be estimated from the total mass profile derived under the assumption of hydrostatic equilibrium (HE), the present sample contains clusters in a wide variety of dynamical states and consequently the HE assumption may not be valid in all cases (see the discussion in Pratt et al. 2007). Instead we estimate $R_{500}$ using $Y_{\mathrm{X}}$ as a mass proxy. This quantity, defined as the product of $M_{\mathrm{g}, 500}$, the gas mass within $R_{500}$, and the spectroscopic temperature in the [0.15-1] $R_{500}$ region, is the X-ray analogue of the integrated SZ signal $Y_{\mathrm{SZ}}$, and has been shown to be a low scatter mass proxy in the numerical simulations of Kravtsov et al. (2006) even in the presence of significant dynamical activity. Recent observational investigations using a variety of cluster samples have demonstrated that $Y_{\mathrm{X}}$ is indeed a low-scatter mass proxy (Maughan 2007; Arnaud et al. 2007), and the theoretical results have been verified in independent numerical simulations (Poole et al. 2007; Yang et al. 2008). We estimate $R_{500}$ iteratively from the $M_{500}-Y_{\mathrm{X}}$ relation derived from XMM-Newton observations of a sample of 10 nearby morphologically relaxed local clusters by Arnaud et al. (2007), viz.,

$h(z)^{2 / 5} M_{500}=10^{14.556 \pm 0.015}\left[\frac{Y_{\mathrm{X}}}{2 \times 10^{14} M_{\odot} \mathrm{keV}}\right]^{0.548 \pm 0.027} h_{70}^{-1} M_{\odot}$,

which was derived using substantially similar methods to those described in this paper. The REXCESS gas density profiles from which $M_{\text {gas }}$ is derived are discussed in Croston et al. (2008).

Note that there is an $\sim 8$ percent normalisation offset of the observed relation when compared to the relation derived by Nagai et al. (2007) from numerical simulations. However, an iterative measurement of $R_{500}$ from the simulated $M_{500}-Y_{\mathrm{X}}$ relation changes the values of the temperature and luminosity by less than 1.5 per cent on average, due to the steep drop of emission with radius. Simulations also suggest a \pm 8 per cent scatter about the $M_{500}-Y_{\mathrm{X}}$. Using randomisation assuming a 1.5 per cent relative change in the measured quantities due to this scatter, we have verified that the slopes and normalisations of the scaling laws do not change, and that the maximum change in the scatter about the relations is only 7 per cent.

\subsection{Luminosities and temperatures}

Bolometric X-ray luminosities, referred to as $L$ throughout the remainder of this paper, were derived for two apertures: (i) the entire cluster emission interior to $R_{500}$ (hereafter $L_{1}$ ); and (ii) in the [0.15-1] $R_{500}$ aperture (hereafter $L_{2}$ ). We estimated the count rates from surface brightness profiles in the [0.3-2] $\mathrm{keV}$ band, and used the best fitting spectral model estimated in the same aperture to convert the count rate to bolometric $([0.01-100] \mathrm{keV})$ luminosity. At $3 \sigma$ significance, the surface brightness profiles are detected out to at least $0.8 R_{500}$ for all clusters. For 11 clusters, we extrapolated the surface brightness profile using a power law with a slope measured from that of the data at large radius. As can be seen in Table 1, in most cases the need for extrapolation is minimal. Measured luminosities for both apertures are given in Table 1. Errors take into account statistical factors, uncertainties in $R_{500}$ and extrapolation uncertainties. These were estimated from Monte Carlo realisations in which the above

\footnotetext{
${ }^{1} M_{500}=500 \rho_{c}(z)(4 \pi / 3) R_{500}^{3}$, where $\rho_{\mathrm{c}}(z)=h^{2}(z) 3 H_{0}^{2} / 8 \pi G$ and $h^{2}(z)=\Omega_{M}(1+z)^{3}+\Omega_{\Lambda}$.
} 
Table 1. Cluster properties.

\begin{tabular}{|c|c|c|c|c|c|c|c|c|c|c|c|}
\hline $\begin{array}{c}\text { Cluster } \\
(1)\end{array}$ & $\begin{array}{c}z \\
(2) \\
\end{array}$ & $\begin{array}{l}T_{1} \\
(3) \\
\end{array}$ & $\begin{array}{l}L_{1} \\
(4) \\
\end{array}$ & $\begin{array}{l}T_{2} \\
(5) \\
\end{array}$ & $\begin{array}{l}L_{2} \\
(6) \\
\end{array}$ & $\begin{array}{l}T_{3} \\
(7) \\
\end{array}$ & $\begin{array}{l}Y_{X} \\
(8) \\
\end{array}$ & $\begin{array}{c}R_{500} \\
(9) \\
\end{array}$ & $\begin{array}{l}R_{\mathrm{det}} \\
(10) \\
\end{array}$ & $\begin{array}{l}\mathrm{CC} \\
(11) \\
\end{array}$ & $\begin{array}{c}\text { Disturbed } \\
\text { (12) }\end{array}$ \\
\hline RXC J0003.8+0203 & 0.0924 & $3.85_{-0.09}^{+0.09}$ & $1.88_{-0.01}^{+0.01}$ & $3.64_{-0.09}^{+0.09}$ & $1.16_{-0.01}^{+0.01}$ & $3.87_{-0.10}^{+0.10}$ & $7.69_{-0.26}^{+0.26}$ & 876.7 & 0.84 & $\cdots$ & 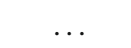 \\
\hline RXC J0006.0-3443 & 0.1147 & $5.03_{-0.19}^{+0.19}$ & $4.13_{-0.05}^{+0.05}$ & $4.60_{-0.16}^{+0.21}$ & $3.18_{-0.05}^{+0.05}$ & $5.18_{-0.20}^{+0.20}$ & $22.74_{-1.21}^{+1.22}$ & 1059.3 & 0.93 & $\ldots$ & $\sqrt{ }$ \\
\hline RXC J0020.7-2542 & 0.1410 & $5.69_{-0.11}^{+0.11}$ & $6.52_{-0.04}^{+0.04}$ & $5.24_{-0.15}^{+0.15}$ & $4.07_{-0.04}^{+0.04}$ & $5.55_{-0.13}^{+0.13}$ & $22.41_{-0.63}^{+0.65}$ & 1045.3 & 1.07 & $\ldots$ & $\ldots$ \\
\hline RXC J0049.4-2931 & 0.1084 & $3.09_{-0.10}^{+0.10}$ & $1.78_{-0.02}^{+0.02}$ & $2.79_{-0.11}^{+0.11}$ & $1.00_{-0.02}^{+0.02}$ & $3.03_{-0.12}^{+0.12}$ & $5.09_{-0.24}^{+0.25}$ & 807.8 & 0.93 & $\ldots$ & .. \\
\hline RXC J0145.0-5300 & 0.1168 & $5.53_{-0.13}^{+0.13}$ & $5.00_{-0.03}^{+0.03}$ & $5.51_{-0.16}^{+0.16}$ & $3.88_{-0.03}^{+0.03}$ & $5.63_{-0.14}^{+0.14}$ & $26.61_{-0.87}^{+0.89}$ & 1089.3 & 1.23 & $\ldots$ & $\sqrt{ }$ \\
\hline RXC J0211.4-4017 & 0.1008 & $2.07_{-0.00}^{+0.07}$ & $0.81_{-0.01}^{+0.01}$ & $2.02_{-0.06}^{+0.06}$ & $0.48_{-0.01}^{+0.01}$ & $2.07_{-0.05}^{+0.05}$ & $2.03_{-0.06}^{+0.06}$ & 685.0 & 1.33 & $\ldots$ & $\ldots$ \\
\hline RXC J0225.1-2928 & 0.0604 & $2.47_{-0.06}^{+0.15}$ & $0.51_{-0.01}^{+0.01}$ & $2.61_{-0.16}^{+0.16}$ & $0.31_{-0.01}^{+0.01}$ & $2.67_{-0.13}^{+0.13}$ & $2.00_{-0.12}^{+0.12}$ & 693.9 & 0.91 & $\ldots$ & $\sqrt{ }$ \\
\hline RXC J0345.7-4112 & 0.0603 & $2.19_{-0.04}^{+0.04}$ & $0.77_{-0.01}^{+0.01}$ & $2.15_{-0.08}^{+0.08}$ & $0.37_{-0.01}^{+0.01}$ & $2.30_{-0.06}^{+0.09}$ & $1.91_{-0.06}^{+0.09}$ & 688.4 & 0.89 & $\sqrt{ }$ & $\ldots$ \\
\hline RXC J0547.6-3152 & 0.1483 & $6.02_{-0.11}^{+0.11}$ & $8.97_{-0.04}^{+0.04}$ & $5.68_{-0.11}^{+0.11}$ & $5.76_{-0.04}^{+0.04}$ & $6.06_{-0.14}^{+0.14}$ & $35.54_{-0.99}^{+1.02}$ & 1133.7 & 1.32 & $\ldots$ & $\ldots$ \\
\hline RXC J0605.8-3518 & 0.1392 & $4.56_{-0.05}^{+0.05}$ & $9.54_{-0.04}^{+0.04}$ & $4.81_{-0.12}^{+0.12}$ & $4.26_{-0.04}^{+0.04}$ & $4.91_{-0.11}^{+0.11}$ & $22.39_{-0.63}^{+0.66}$ & 1045.9 & 1.17 & $\sqrt{ }$ & $\ldots$ \\
\hline RXC J0616.8-4748 & 0.1164 & $4.22_{-0.10}^{+0.10}$ & $2.38_{-0.02}^{+0.02}$ & $4.16_{-0.12}^{+0.12}$ & $1.88_{-0.02}^{+0.02}$ & $4.17_{-0.11}^{+0.11}$ & $11.81_{-0.41}^{+0.39}$ & 939.2 & 1.12 & $\ldots$ & $\sqrt{ }$ \\
\hline RXC J0645.4-5413 & 0.1644 & $6.95_{-0.13}^{+0.13}$ & $18.88_{-0.10}^{+0.10}$ & $6.97_{-0.19}^{+0.19}$ & $11.39_{-0.09}^{+0.09}$ & $7.27_{-0.18}^{+0.18}$ & $71.61_{-2.33}^{+2.35}$ & 1280.0 & 1.28 & $\ldots$ & $\ldots$ \\
\hline RXC J0821.8+0112 & 0.0822 & $2.68_{-0.09}^{+0.09}$ & $0.77_{-0.01}^{+0.01}$ & $2.44_{-0.12}^{+0.12}$ & $0.54_{-0.01}^{+0.01}$ & $2.84_{-0.10}^{+0.10}$ & $3.34_{-0.15}^{+0.15}$ & 755.9 & 0.93 & $\ldots$ & $\ldots$ \\
\hline RXC J0958.3-1103 & 0.1669 & $5.34_{-0.21}^{+0.21}$ & $11.56_{-0.15}^{+0.15}$ & $5.85_{-0.40}^{+0.45}$ & $5.25_{-0.16}^{+0.16}$ & $6.30_{-0.44}^{+0.50}$ & $28.04_{-2.30}^{+2.67}$ & 1077.4 & 0.78 & $\sqrt{ }$ & $\ldots$ \\
\hline RXC J1044.5-0704 & 0.1342 & $3.41_{-0.03}^{+0.03}$ & $7.42_{-0.02}^{+0.02}$ & $3.52_{-0.05}^{+0.05}$ & $3.00_{-0.02}^{+0.02}$ & $3.57_{-0.05}^{+0.05}$ & $11.77_{-0.19}^{+0.19}$ & 931.9 & 1.09 & $\sqrt{ }$ & $\ldots$ \\
\hline RXC J1141.4-1216 & 0.1195 & $3.31_{-0.03}^{+0.03}$ & $3.75_{-0.01}^{+0.01}$ & $3.40_{-0.06}^{+0.06}$ & $1.70_{-0.01}^{+0.01}$ & $3.54_{-0.05}^{+0.05}$ & $8.60_{-0.15}^{+0.16}$ & 885.2 & 1.25 & $\sqrt{ }$ & $\cdots$ \\
\hline RXC J1236.7-3354 & 0.0796 & $2.70_{-0.05}^{+0.05}$ & $1.03_{-0.01}^{+0.01}$ & $2.57_{-0.03}^{+0.11}$ & $0.61_{-0.01}^{+0.01}$ & $2.73_{-0.01}^{+0.09}$ & $3.27_{-0.02}^{+0.15}$ & 753.5 & 0.99 & $\ldots$ & $\ldots$ \\
\hline RXC J1302.8-0230 & 0.0847 & $2.97_{-0.07}^{+0.06}$ & $1.38_{-0.01}^{+0.01}$ & $2.92_{-0.07}^{+0.09}$ & $0.83_{-0.01}^{+0.01}$ & $3.44_{-0.07}^{+0.07}$ & $6.07_{-0.18}^{+0.19}$ & 842.1 & 1.22 & $\sqrt{ }$ & $\sqrt{ }$ \\
\hline RXC J1311.4-0120 & 0.1832 & $8.91_{-0.08}^{+0.08}$ & $36.06_{-0.08}^{+0.08}$ & $8.24_{-0.13}^{+0.13}$ & $15.13_{-0.07}^{+0.07}$ & $8.44_{-0.12}^{+0.12}$ & $88.18_{-1.50}^{+1.51}$ & 1319.2 & 1.31 & $\sqrt{ }$ & $\ldots$ \\
\hline RXC J1516.3+0005 & 0.1181 & $4.51_{-0.06}^{+0.06}$ & $4.12_{-0.02}^{+0.02}$ & $4.18_{-0.08}^{+0.08}$ & $2.77_{-0.02}^{+0.02}$ & $4.48_{-0.07}^{+0.07}$ & $15.81_{-0.31}^{+0.30}$ & 989.9 & 1.29 & $\ldots$ & $\ldots$ \\
\hline RXC J1516.5-0056 & 0.1198 & $3.55_{-0.07}^{+0.07}$ & $2.31_{-0.02}^{+0.02}$ & $3.40_{-0.08}^{+0.08}$ & $1.77_{-0.02}^{+0.02}$ & $3.74_{-0.09}^{+0.10}$ & $11.08_{-0.36}^{+0.41}$ & 927.0 & 1.37 & $\ldots$ & $\sqrt{ }$ \\
\hline RXC J2014.8-2430 & 0.1538 & $4.78_{-0.05}^{+0.05}$ & $21.06_{-0.07}^{+0.07}$ & $5.63_{-0.11}^{+0.11}$ & $7.52_{-0.07}^{+0.07}$ & $5.73_{-0.10}^{+0.10}$ & $39.89_{-0.82}^{+0.78}$ & 1155.3 & 1.09 & $\sqrt{ }$ & $\ldots$ \\
\hline RXC J2023.0-2056 & 0.0564 & $2.71_{-0.09}^{+0.09}$ & $0.61_{-0.01}^{+0.01}$ & $2.46_{-0.12}^{+0.12}$ & $0.40_{-0.01}^{+0.01}$ & $2.72_{-0.09}^{+0.09}$ & $2.81_{-0.12}^{+0.13}$ & 739.5 & 0.86 & $\ldots$ & $\sqrt{ }$ \\
\hline RXC J2048.1-1750 & 0.1475 & $4.65_{-0.07}^{+0.13}$ & $5.13_{-0.03}^{+0.03}$ & $4.59_{-0.08}^{+0.08}$ & $4.40_{-0.03}^{+0.03}$ & $5.01_{-0.11}^{+0.11}$ & $26.91_{-0.80}^{+0.81}$ & 1078.0 & 1.48 & $\ldots$ & $\sqrt{ }$ \\
\hline RXC J2129.8-5048 & 0.0796 & $3.81_{-0.15}^{+0.15}$ & $1.46_{-0.02}^{+0.02}$ & $3.64_{-0.12}^{+0.16}$ & $1.19_{-0.02}^{+0.02}$ & $3.88_{-0.14}^{+0.14}$ & $8.67_{-0.41}^{+0.40}$ & 900.6 & 0.93 & $\ldots$ & $\sqrt{ }$ \\
\hline RXC J2149.1-3041 & 0.1184 & $3.26_{-0.04}^{+0.04}$ & $3.56_{-0.02}^{+0.02}$ & $3.40_{-0.08}^{+0.08}$ & $1.58_{-0.02}^{+0.02}$ & $3.50_{-0.07}^{+0.07}$ & $8.65_{-0.32}^{+0.32}$ & 886.6 & 1.26 & $\sqrt{ }$ & $\cdots$ \\
\hline RXC J2157.4-0747 & 0.0579 & $2.46_{-0.08}^{+0.08}$ & $0.45_{-0.01}^{+0.01}$ & $2.30_{-0.06}^{+0.10}$ & $0.37_{-0.01}^{+0.01}$ & $2.76_{-0.07}^{+0.07}$ & $3.07_{-0.11}^{+0.11}$ & 751.5 & 0.97 & $\ldots$ & $\sqrt{ }$ \\
\hline RXC J2217.7-3543 & 0.1486 & $4.86_{-0.09}^{+0.09}$ & $6.12_{-0.03}^{+0.03}$ & $4.45_{-0.09}^{+0.09}$ & $3.70_{-0.03}^{+0.03}$ & $4.65_{-0.08}^{+0.10}$ & $20.32_{-0.47}^{+0.54}$ & 1022.6 & 1.33 & $\ldots$ & $\ldots$ \\
\hline RXC J2218.6-3853 & 0.1411 & $5.84_{-0.11}^{+0.11}$ & $9.43_{-0.06}^{+0.06}$ & $5.88_{-0.15}^{+0.20}$ & $5.60_{-0.06}^{+0.06}$ & $6.16_{-0.19}^{+0.19}$ & $34.36_{-1.33}^{+1.30}$ & 1130.1 & 1.04 & $\cdots$ & $\sqrt{ }$ \\
\hline RXC J2234.5-3744 & 0.1510 & $7.78_{-0.15}^{+0.15}$ & $19.15_{-0.11}^{+0.11}$ & $6.95_{-0.14}^{+0.14}$ & $12.36_{-0.10}^{+0.10}$ & $7.30_{-0.12}^{+0.12}$ & $70.43_{-1.54}^{+1.51}$ & 1283.2 & 1.15 & $\ldots$ & $\cdots$ \\
\hline RXC J2319.6-7313 & 0.0984 & $2.22_{-0.03}^{+0.03}$ & $2.00_{-0.02}^{+0.02}$ & $2.48_{-0.08}^{+0.08}$ & $0.97_{-0.01}^{+0.01}$ & $2.52_{-0.07}^{+0.07}$ & $4.37_{-0.16}^{+0.16}$ & 788.7 & 1.11 & $\sqrt{ }$ & $\sqrt{ }$ \\
\hline
\end{tabular}

The luminosity is the bolometric [0.01-100] keV luminosity. All quantities are calculated assuming $\Omega_{M}=0.3, \Omega_{\Lambda}=0.7$, and $h_{0}=0.7$. Columns: (1) Cluster name; (2) z: cluster redshift; (3) $T_{1}$ : spectroscopic temperature of the $R<R_{500}$ region in keV; (4) $L_{1}$ : luminosity in the $R<R_{500}$ region in units of $10^{44} \mathrm{erg} \mathrm{s}^{-1}$; (5) $T_{2}$ : spectroscopic temperature in the $[0.15-1] R_{500}$ region in keV; (6) $L_{2}$ : luminosity in the $[0.15-1] R_{500}$ region in units of $10^{44} \mathrm{erg} \mathrm{s}^{-1}$; (7) $T_{3}$ : spectroscopic temperature in the [0.15-0.75] $R_{500}$ region in keV; (8) $Y_{\mathrm{X}}$ in units of $10^{13} M_{\odot} \mathrm{keV}$; (9) $R_{500}$ in kpc; (10) ratio of the detection radius of the surface brightness profile at $3 \sigma$ significance, $R_{\text {det }}$, to $R_{500}$; (11) systems classified as cool cores on the basis of central density vs. cooling time (see Sect. 2.3); (12) systems classified as disturbed on the basis of the centre shift parameter $\langle w\rangle$ (see Sect. 2.3).

procedure, including extrapolation, was repeated for 100 surface brightness profiles, the profiles and $R_{500}$ values each being randomised according to the observed uncertainties. A PSF correction was implemented by using the gas density profile derived from regularised deprojection of the surface brightness as described in Croston et al. (2006); the correction was obtained from the ratio of the observed to PSF-corrected count rates in each aperture. The correction is negligible in the full aperture but can be up to 13 per cent for strong cooling core systems in the core-excluded aperture.

We have also calculated luminosities in the [0.1-2.4] keV and [0.5-2] keV bands for ease of comparison with previous soft X-ray survey results from ROSAT. Luminosities and their associated scaling relations are given in Appendix B.
Spectroscopic temperatures were measured in the $[0.15-$ 0.75] $R_{500}$ aperture $^{2}$ (referred to hereafter as $T_{3}$ ) from iteration about the $M_{500}-Y_{\mathrm{X}}$ relation of Arnaud et al. (2007). Temperatures in the full aperture and in the [0.15-1] $R_{500}$ aperture (hereafter $T_{1}$ and $T_{2}$, respectively) were then calculated by re-extraction of spectra in the relevant regions. In all cases the spectra were fitted with a MEKAL model with an absorption fixed at the HI value (excepting RXC J2014.82430, which was found to have a significantly higher absorption than that indicated from the HI value). The three EPIC cameras were fitted simultaneously in the [0.3-10] keV band, with the regions around the instrumental lines $(1.4-1.6 \mathrm{keV}$ for

\footnotetext{
2 Note that this aperture is more appropriate for comparison with distant clusters, which often have poor signal to noise in the outer regions.
} 
Table 2. Observed bolometric X-ray luminosity scaling relations.

\begin{tabular}{|c|c|c|c|c|c|c|}
\hline \multirow[t]{3}{*}{ Relation } & \multicolumn{6}{|c|}{ Fitting method } \\
\hline & \multicolumn{3}{|c|}{$\operatorname{BCES}(\mathrm{Y} \mid \mathrm{X})$} & \multicolumn{3}{|c|}{ BCES Orthogonal } \\
\hline & $C\left(10^{44} \mathrm{erg} \mathrm{s}^{-1}\right)$ & $\bar{\alpha}$ & $\sigma_{\ln \mathrm{L}, \text { intrinsic }}$ & $C\left(10^{44} \mathrm{erg} \mathrm{s}^{-1}\right)$ & $\alpha$ & $\sigma_{\ln \mathrm{L}, \text { intrinsic }}$ \\
\hline \multirow{2}{*}{\multicolumn{7}{|c|}{$R<R_{500}$}} \\
\hline & & & & & & \\
\hline$L_{1}-T_{1}$ & $6.07 \pm 0.58$ & $2.70 \pm 0.24$ & $0.663 \pm 0.116$ & $7.13 \pm 1.03$ & $3.35 \pm 0.32$ & $0.733 \pm 0.135$ \\
\hline$L_{1}-T_{3}$ & $5.62 \pm 0.46$ & $2.88 \pm 0.23$ & $0.525 \pm 0.097$ & $6.27 \pm 0.67$ & $3.42 \pm 0.27$ & $0.560 \pm 0.115$ \\
\hline$L_{1}-Y_{\mathrm{X}}$ & $5.20 \pm 0.36$ & $0.99 \pm 0.05$ & $0.384 \pm 0.060$ & $5.35 \pm 0.38$ & $1.04 \pm 0.06$ & $0.383 \pm 0.061$ \\
\hline$L_{1}-M_{Y}$ & $1.81 \pm 0.13$ & $1.81 \pm 0.10$ & $a \ldots$ & $1.74 \pm 0.13$ & $1.96 \pm 0.11$ & $a \ldots$ \\
\hline$L_{1}-M_{Y} \mathrm{MB}^{b}$ & $1.45 \pm 0.12$ & $1.90 \pm 0.11$ & ${ }^{a} \ldots$ & $1.38 \pm 0.12$ & $2.08 \pm 0.13$ & ${ }^{a} \ldots$ \\
\hline \multicolumn{7}{|l|}{ Cool core } \\
\hline$L_{1}-T_{1}$ & $11.15 \pm 2.42$ & $2.71 \pm 0.48$ & $0.432 \pm 0.108$ & $12.79 \pm 3.80$ & $3.15 \pm 0.63$ & $0.479 \pm 0.135$ \\
\hline$L_{1}-Y_{\mathrm{X}}$ & $7.71 \pm 0.58$ & $1.04 \pm 0.07$ & $0.234 \pm 0.103$ & $7.84 \pm 0.65$ & $1.06 \pm 0.09$ & $0.236 \pm 0.107$ \\
\hline \multicolumn{7}{|l|}{ Non-cool core } \\
\hline$L_{1}-T_{1}$ & $4.78 \pm 0.29$ & $2.89 \pm 0.21$ & $0.267 \pm 0.058$ & $4.97 \pm 0.29$ & $3.06 \pm 0.19$ & $0.285 \pm 0.068$ \\
\hline$L_{1}-Y_{\mathrm{X}}$ & $4.27 \pm 0.20$ & $0.96 \pm 0.05$ & $0.214 \pm 0.035$ & $4.32 \pm 0.20$ & $0.98 \pm 0.05$ & $0.214 \pm 0.036$ \\
\hline \multicolumn{7}{|l|}{ Disturbed } \\
\hline$L_{1}-T_{1}$ & $4.18 \pm 0.59$ & $2.49 \pm 0.56$ & $0.497 \pm 0.215$ & $5.43 \pm 2.74$ & $3.19 \pm 0.78$ & $0.646 \pm 0.346$ \\
\hline$L_{1}-Y_{\mathrm{X}}$ & $3.72 \pm 0.27$ & $0.92 \pm 0.09$ & $0.245 \pm 0.120$ & $3.85 \pm 0.32$ & $0.96 \pm 0.08$ & $0.249 \pm 0.123$ \\
\hline \multicolumn{7}{|l|}{ Regular } \\
\hline$L_{1}-T_{1}$ & $7.26 \pm 0.86$ & $2.62 \pm 0.21$ & $0.578 \pm 0.118$ & $7.97 \pm 1.28$ & $3.13 \pm 0.33$ & $0.634 \pm 0.142$ \\
\hline$L_{1}-Y_{\mathrm{X}}$ & $6.15 \pm 0.42$ & $0.97 \pm 0.05$ & $0.302 \pm 0.058$ & $6.21 \pm 0.44$ & $1.00 \pm 0.05$ & $0.303 \pm 0.059$ \\
\hline & & & $0.15<$ & $<R_{500}$ & & \\
\hline \multicolumn{7}{|c|}{ 过 } \\
\hline$L_{2}-T_{2}$ & $3.89 \pm 0.18$ & $2.78 \pm 0.13$ & $0.269 \pm 0.055$ & $4.06 \pm 0.22$ & $2.94 \pm 0.15$ & $0.279 \pm 0.059$ \\
\hline$L_{2}-T_{3}$ & $3.31 \pm 0.16$ & $2.84 \pm 0.17$ & $0.331 \pm 0.068$ & $3.48 \pm 0.21$ & $3.07 \pm 0.18$ & $0.346 \pm 0.075$ \\
\hline$L_{2}-Y_{\mathrm{X}}$ & $3.05 \pm 0.07$ & $0.97 \pm 0.03$ & $0.156 \pm 0.038$ & $3.06 \pm 0.07$ & $0.98 \pm 0.03$ & $0.156 \pm 0.038$ \\
\hline$L_{2}-M_{Y}$ & $1.09 \pm 0.05$ & $1.77 \pm 0.05$ & ${ }^{a} \ldots$ & $1.08 \pm 0.04$ & $1.80 \pm 0.05$ & ${ }^{a} \ldots$ \\
\hline \multicolumn{7}{|l|}{ Cool core } \\
\hline$L_{2}-T_{2}$ & $4.31 \pm 0.42$ & $2.58 \pm 0.23$ & $0.242 \pm 0.110$ & $4.46 \pm 0.56$ & $2.70 \pm 0.26$ & $0.247 \pm 0.113$ \\
\hline$L_{2}-Y_{\mathrm{X}}$ & $3.36 \pm 0.16$ & $0.96 \pm 0.04$ & $0.144 \pm 0.098$ & $3.38 \pm 0.17$ & $0.97 \pm 0.05$ & $0.145 \pm 0.098$ \\
\hline \multicolumn{7}{|c|}{ Non-cool core } \\
\hline$L_{2}-T_{2}$ & $3.74 \pm 0.21$ & $2.89 \pm 0.18$ & $0.231 \pm 0.035$ & $3.88 \pm 0.22$ & $3.02 \pm 0.19$ & $0.237 \pm 0.039$ \\
\hline$L_{2}-Y_{\mathrm{X}}$ & $2.91 \pm 0.06$ & $0.97 \pm 0.03$ & $0.114 \pm 0.027$ & $2.92 \pm 0.06$ & $0.98 \pm 0.03$ & $0.114 \pm 0.027$ \\
\hline \multicolumn{7}{|l|}{ Disturbed } \\
\hline$L_{2}-T_{2}$ & $3.58 \pm 0.41$ & $2.88 \pm 0.37$ & $0.295 \pm 0.080$ & $4.00 \pm 0.73$ & $3.18 \pm 0.38$ & $0.312 \pm 0.090$ \\
\hline$L_{2}-Y_{\mathrm{X}}$ & $2.77 \pm 0.07$ & $0.99 \pm 0.04$ & $0.111 \pm 0.096$ & $2.79 \pm 0.08$ & $0.99 \pm 0.04$ & $0.111 \pm 0.096$ \\
\hline \multicolumn{7}{|l|}{ Regular } \\
\hline$L_{2}-T_{2}$ & $4.13 \pm 0.21$ & $2.68 \pm 0.11$ & $0.225 \pm 0.070$ & $4.20 \pm 0.23$ & $2.76 \pm 0.11$ & $0.231 \pm 0.075$ \\
\hline$L_{2}-Y_{\mathrm{X}}$ & $3.24 \pm 0.08$ & $0.94 \pm 0.02$ & $0.115 \pm 0.045$ & $3.24 \pm 0.08$ & $0.94 \pm 0.02$ & $0.115 \pm 0.045$ \\
\hline
\end{tabular}

Each set of observables $(L, A)$ is fitted with a power law relation of the form $h(z)^{n} L=C\left(A / A_{0}\right)^{\alpha}$, with $A_{0}=5 \mathrm{keV}, 2 \times 10^{14} M_{\odot} \mathrm{keV}$ and $2 \times 10^{14} M_{\odot}$, and $n=-1,-9 / 5$ and $-7 / 3$ for $T, Y_{\mathrm{X}}$ and $M$, respectively. Results are given for the BCES (Y|X) and BCES orthogonal fitting methods (see Sect. 2.4). ${ }^{a}$ Since $M$ is derived from $Y_{\mathrm{X}}$, the values of the scatter in the $L-M$ relation are identical to those for the $L-Y_{\mathrm{X}}$ relation; ${ }^{b}$ corrected for Malmquist bias (see Appendix B). $L_{1} / T_{1}$ : luminosity/temperature interior to $R_{500} ; L_{2} / T_{2}$ : luminosity/temperature in the [0.15-1] $R_{500}$ aperture; $T_{3}$ : temperature in the [0.15-0.75] $R_{500}$ aperture; $M_{Y}$ : mass measured from the $M_{500}-Y_{\mathrm{X}}$ relation of Arnaud et al. (2007).

all cameras and 7.45-9 $\mathrm{keV}$ for the pn camera) excluded from the fit. Temperatures for all three apertures are listed in Table 1.

\subsection{Subsamples}

We further subdivide the sample to elucidate the effects of cool cores and merger-related phenomena on the scaling relations.

\subsubsection{Cooling time classification}

In an approach similar to that used by O'Hara et al. (2006), we use gas density and cooling time profiles to classify cooling core systems. Croston et al. (2008) describe the gas density and cooling time profiles of the present sample, which are fully deprojected and PSF-corrected using the non-parametric method described in Croston et al. (2006). We estimate the central gas density $n_{e, 0}$ from a $\beta$ model fit to the deconvolved, deprojected 

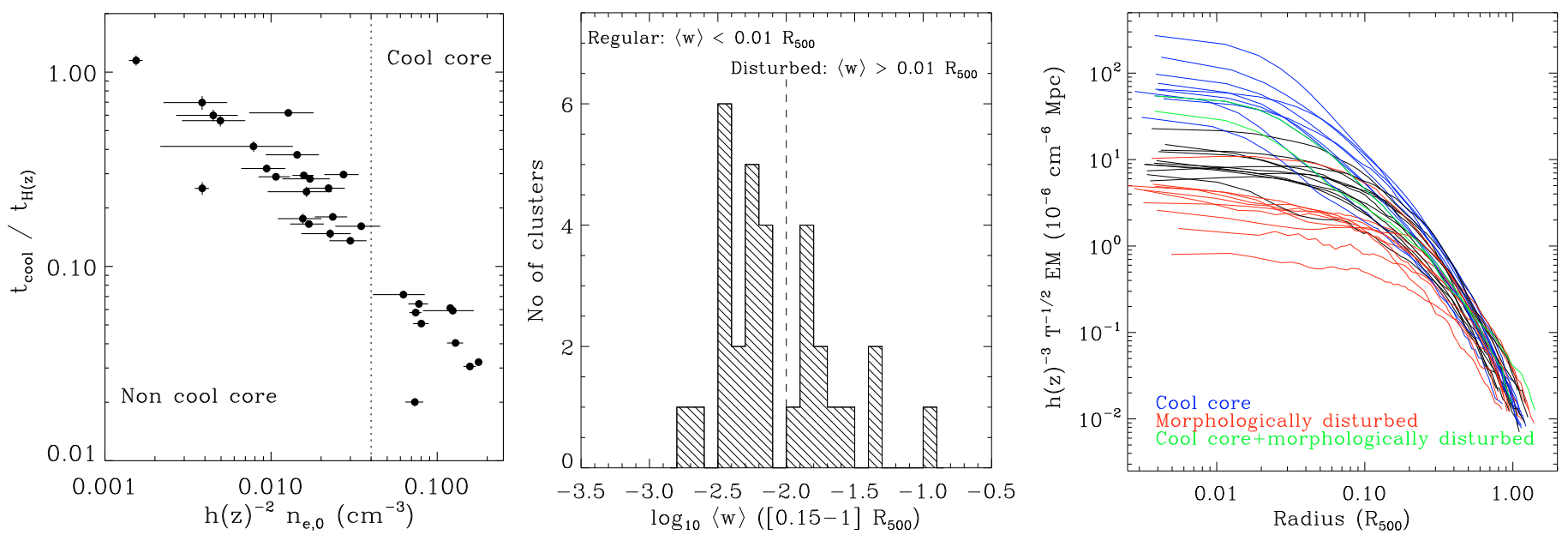

Fig. 1. Definition of cluster subsamples. Left: central cooling time vs. central gas density, $n_{e, 0}$. The dotted line delineates the threshold we use to define cool core systems: $h(z)^{-2} n_{e, 0}>4 \times 10^{-2} \mathrm{~cm}^{-3}, t_{\text {cool }}<10^{9}$ years. Centre: histogram of centre shift parameter $\langle w\rangle$, evaluated in the [0.15-1] $R_{500}$ aperture. Clusters with $\langle w\rangle>0.01 R_{500}$ are classified as morphologically disturbed. Right: emission measure profiles of the REXCESS sample, scaled according to the standard dependence on temperature and expected evolution with redshift. Systems classified as cool core and as morphologically disturbed are indicated (see Sect. 2.3).

gas density profiles interior to $0.03 R_{500}$. Figure 1 shows the central cooling time versus $n_{e, 0}$, which exhibits a strong correlation, as expected since the cooling time is derived from the gas density. We classify clusters according to their central gas density $n_{e, 0}$, such that those with $h(z)^{-2} n_{e, 0}>4 \times 10^{-2} \mathrm{~cm}^{-3}$ (equivalent to those with a central $E M$ value $E M_{\text {cen }} \gtrsim 20 \times 10^{-6} \mathrm{~cm}^{-6} \mathrm{Mpc}$ in the right hand panel of Fig. 1) are defined as cool core systems; 10/31 clusters are classified as such. Figure 1 shows that these systems have central cooling times $t_{\mathrm{cool}, 0}<10^{9}$ years.

\subsubsection{Morphological classification}

The sample also contains clusters in a wide variety of dynamical states (Böhringer et al. 2007; Pratt et al. 2007). To investigate the effect of dynamical state on the relations, we have calculated values of centroid shift $\langle w\rangle$, defined as the standard deviation of the projected separations between the X-ray peak and the centroid at each radius in the $[0.1-1] R_{500}$ region:

$\langle w\rangle=\left[\frac{1}{N-1} \sum\left(\Delta_{i}-\langle\Delta\rangle\right)^{2}\right]^{1 / 2} \times \frac{1}{R_{500}}$,

where $\Delta_{i}$ is the projected distance between the X-ray peak and centroid in the $i$ th aperture.

Introduced by Mohr et al. (1993), this quantity was found to be the most sensitive indicator of dynamical activity in the numerical simulations of Poole et al. (2007). We calculate centroids in circular apertures of radii $n \times 0.1 \times R_{500}$ with $n=2,3 \ldots 10$, excluding the central regions to avoid biases associated with enhanced emission from cool cores (although exclusion of the central region does not have a significant effect on the results). The centroid shift $\langle w\rangle$ is then defined as the standard deviation of the projected separations between the X-ray surface brightness peak and the centroid in units of $R_{500}$. A forthcoming paper will discuss these results in more detail. For the current analysis, the distribution of $\langle w\rangle$ for the present sample is shown in the central panel of Fig. 1 . We classify clusters with $\langle w\rangle>0.01 R_{500}$ as morphologically disturbed, and clusters with $\langle w\rangle<0.01 R_{500}$ as morphologically regular. In total, $12 / 31$ clusters are defined as morphologically disturbed.

The different subsample classifications are indicated in Table 1 and illustrated in Fig. 1. In general, the presence of a cool core is anti-correlated with indications for morphological disturbance. However, two clusters possess both a cool core and display evidence for morphological disturbance (RXC J1302.8 0230 and RXC J2319.6 -7313). A gallery of the cool core and non-cool core systems, sorted by $\langle w\rangle$, can be found in Figs. A.1 and A.2, respectively, in Appendix A.

\subsection{Fitting procedure}

For each set of observables $(B, A)$, we fitted a power law relation of the form $h(z)^{n} B=C\left(A / A_{0}\right)^{\alpha}$, where $h(z)$ is the Hubble constant normalised to its present day value and $n$ was fixed to the expected scaling with $z$. The fit was undertaken using linear regression in the log-log plane, taking the uncertainties in both variables into account. Assuming a linear relation of the form $Y=a X+b$, and a sample of $N$ data points $\left(Y_{i}, X_{i}\right)$ with errors $\sigma_{Y_{i}}$ and $\sigma_{X_{i}}$, the raw scatter was estimated using the error weighted orthogonal distances to the regression line:

$$
\sigma_{\text {raw }}^{2}=\frac{1}{N-2} \sum_{i=1}^{N} w_{i}\left(Y_{i}-a X_{i}-b\right)^{2}
$$

where

$$
w_{i}=\frac{1 / \sigma_{i}^{2}}{(1 / N) \sum_{i=1}^{N} 1 / \sigma_{i}^{2}} \quad \text { and } \quad \sigma_{i}^{2}=\sigma_{Y_{i}}^{2}+a^{2} \sigma_{X_{i}}^{2} .
$$

The intrinsic scatter was computed from the quadratic difference between the raw scatter and that expected from the statistical uncertainties.

As Figs. 2-5 show, the uncertainties in the present data set are entirely negligible compared to the intrinsic scatter, so that error weighting of individual data points will have no effect on the resulting fits. In the following we use the BCES regression method (Akritas \& Bershady 1996), which takes into account measurement errors in both coordinates and intrinsic scatter in the data and is widely used in astronomical regression, giving results that may easily be compared with other data sets fitted using the same method.

It is well-known that different regression methods give different slopes even at the population level (e.g., Isobe et al. 1990; 

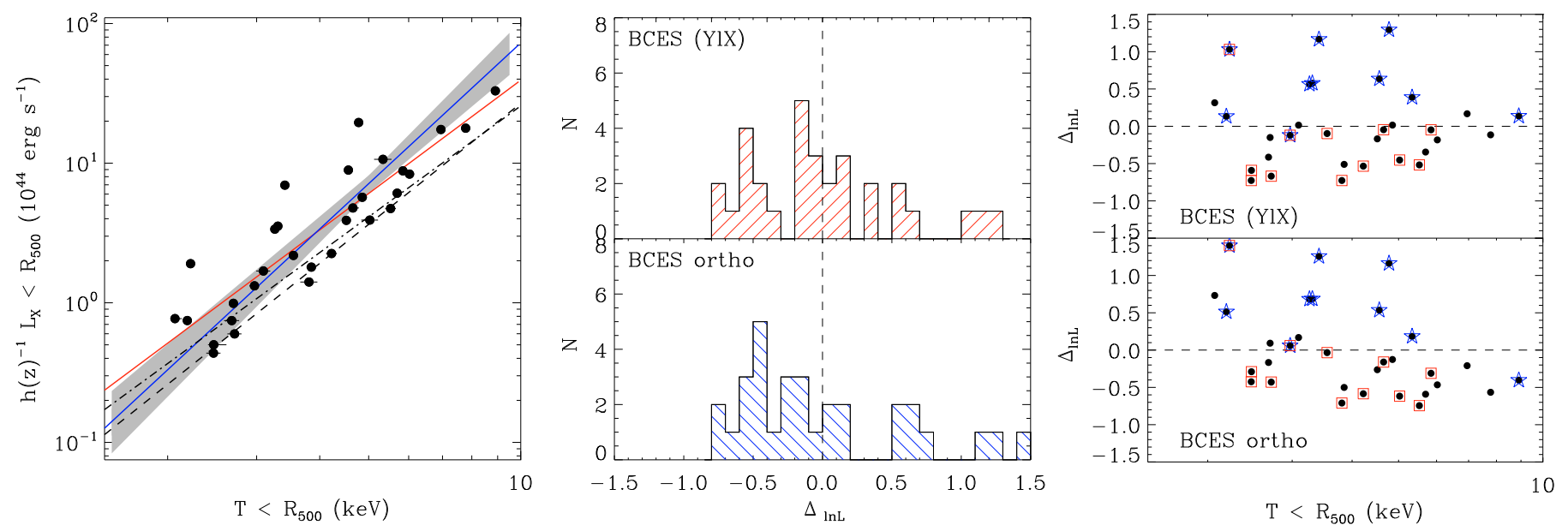

Fig. 2. Left: $L_{1}-T_{1}$ relation for the REXCESS sample (quantities derived from all emission interior to $R_{500}$ ). The error bars are smaller than the points in many cases. The best fitting power law relation derived from the BCES (Y|X) (red line) and BCES orthogonal (blue line) are overplotted; the shaded region corresponds to the $1 \sigma$ uncertainty on the latter. The dashed and dot-dashed lines are the relations of Arnaud \& Evrard (1999) and Markevitch (1998), respectively. Centre: histogram of the log space residuals from the best fitting $L-T$ relation, derived from each fitting method as indicated. Right: Log space residuals for both fitting methods as indicated. Cooling core clusters (blue stars) and morphologically disturbed clusters (red squares) occupy two distinct regions in the plot in both cases.
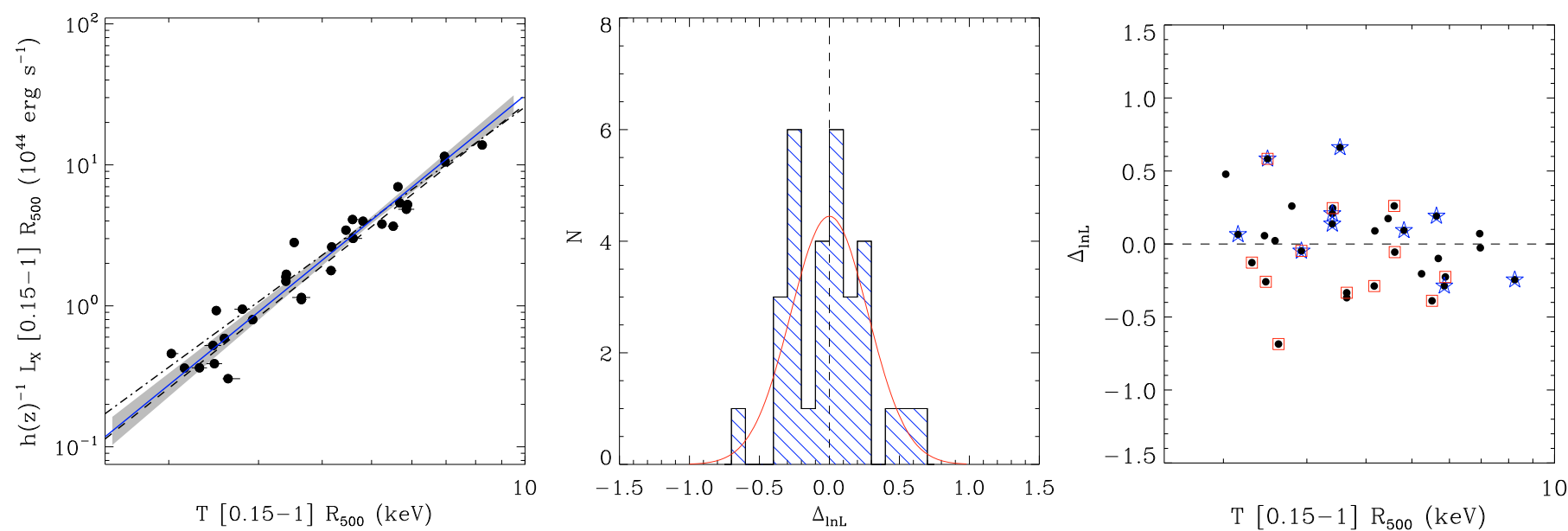

Fig. 3. Left: $L_{2}-T_{2}$ relation for the REXCESS sample (quantities derived from emission in the $0.15 R_{500}<R<R_{500}$ aperture). The best fitting power law relation derived from the BCES orthogonal fitting method is overplotted as a solid line (the BCES (Y|X) results are very similar); the shaded region corresponds to the $1 \sigma$ uncertainty on the fit. The dashed and dot-dashed lines are the relations of Arnaud \& Evrard (1999) and Markevitch (1998), respectively. Centre: histogram of the log space residuals from the best fitting $L-T$ relation, derived from the BCES orthogonal fit method. The solid curve is a Gaussian with $\sigma_{\ln L}=0.28$, corresponding to the scatter about the best fitting relation. Right: Log space residuals. Cooling core clusters (blue stars) and morphologically disturbed clusters (red squares) are less obviously segregated once the central region is excised.

Akritas \& Bershady 1996). It is therefore of paramount importance to choose the regression method best suited to the data in hand. With the present data set, there is no easy answer to the question of which quantity to treat as the dependent variable and which to treat as the independent variable. In cosmological and theoretical applications, the mass of a cluster is its most fundamental property. Given the tight mass-temperature relation (e.g., Arnaud et al. 2005), it is reasonable to assume that $T$ is closely coupled to the mass. However, as will be seen below, there is a large intrinsic scatter in $L$, presumably due to baryon physics. One possible minimisation method would thus treat $L$ as the dependent variable. A second possible minimisation method would be to assume that both variables are quasi-independent, and to treat them symmetrically.

In the following, we thus give the results from the BCES (Y|X) fitting method, which minimises the residuals in $L$, and from the BCES orthogonal fitting method, which minimises the squared orthogonal distances. In the case of maximum scatter (the raw, uncorrected $L_{1}-T_{1}$ relation), the BCES (Y|X) method typically gives slightly shallower slopes than the orthogonal BCES method ${ }^{3}$. As the scatter decreases, the various regression methods give results which agree very well within their $1 \sigma$ uncertainties (Table 2). Uncertainties on all fit parameters and associated scatter are determined from 10000 bootstrap resamples of the data. Since measurement errors are at the 1-3 per cent level, we give only estimates of the intrinsic dispersion about the best fitting relations.

3 The BCES (Y|X) method gives precisely the same results as the modified weighted least squares (WLSS) method described in Pratt et al. (2006). 

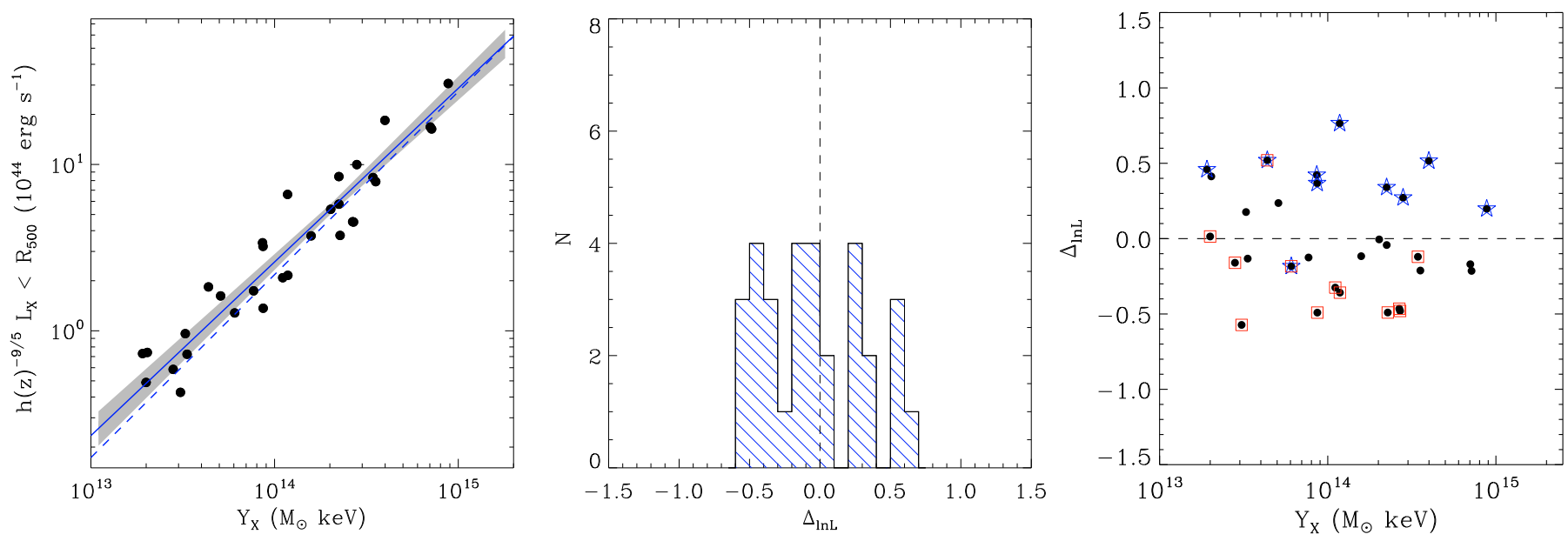

Fig. 4. Left: $L_{1}-Y_{\mathrm{X}}$ relation for the REXCESS sample, with luminosity derived from all emission interior to $R_{500}$. The error bars are smaller than the points in many cases. The best fitting power law relation derived from the BCES orthogonal fitting method is overplotted as a solid line (the BCES (Y|X) results are very similar); the shaded region corresponds to the $1 \sigma$ uncertainty on the fit. The dashed line is the fit derived by Maughan (2007) from observations of 115 galaxy clusters in the Chandra archive. The agreement is excellent. Centre: histogram of the log space residuals about the best fitting orthogonal BCES relation. Right: Log space residuals of the different subsets. Blue stars: cooling core clusters; red squares: morphologically disturbed systems.
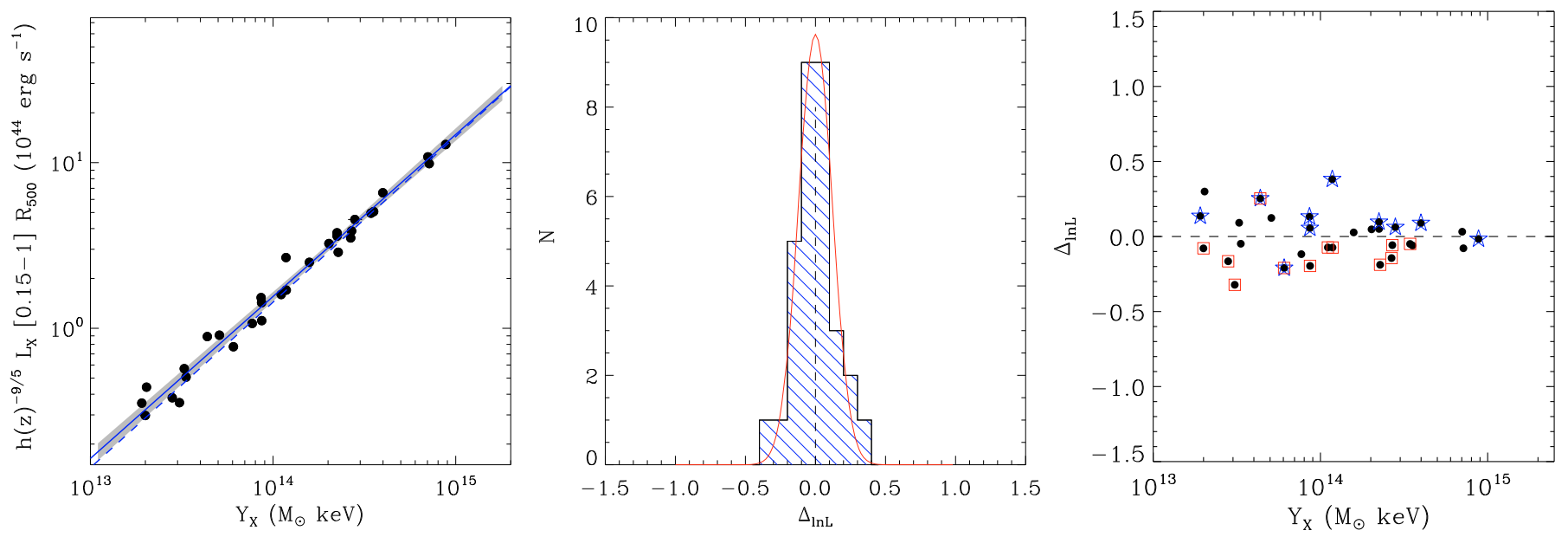

Fig. 5. Left: $L_{2}-Y_{\mathrm{X}}$ relation for the REXCESS sample, with luminosity derived from emission in the $0.15 R_{500}<R<R_{500}$ aperture. The best fitting power law relation derived from the BCES orthogonal fitting method, which takes into account errors in both coordinates and intrinsic scatter in the data, is overplotted as a solid line (the BCES $(\mathrm{Y} \mid \mathrm{X})$ results are very similar); the shaded region corresponds to the $1 \sigma$ uncertainty on the fit. The dashed line is the fit derived by Maughan (2007) from observations of 115 galaxy clusters in the Chandra archive. The agreement is again excellent. Centre: histogram of the log space residuals about the best fitting orthogonal BCES relation. The solid curve is a Gaussian with $\sigma_{\ln L}=0.16$, corresponding to the scatter about the relation. Right: Log space residuals of the different subsets. Blue stars: cooling core clusters, red squares: morphologically disturbed systems.

\section{Results}

\subsection{Scaled emission measure profiles}

The emission measure $(E M)$ was calculated from the surface brightness profiles extracted in the [0.3-2] keV band via:

$E M(r)=\frac{4 \pi(1+z)^{4} S(\theta(x))}{\epsilon(T, z)} ; \quad r=d_{A}(z) \theta$

where $S(\theta)$ is the surface brightness, $d_{A}(z)$ is the angular distance at redshift $z$, and $\epsilon(T, z)$ is the emissivity, which has been calculated taking into account absorption and the instrument response (e.g. Neumann \& Arnaud 1999). We then scaled the EM profiles according to their expected evolution with redshift and dependence on temperature, $E M \propto h(z)^{-3} T^{-1 / 2}$, shown in the right hand panel of Fig. 1. The behaviour of the scaled profiles is very similar to that seen in the gas density profiles discussed in Croston et al. (2008): outside the central regions, the dispersion rapidly decreases and the profiles begin to show indications of similarity. The relative dispersion in scaled profiles shows a broad minimum of $\sigma /\langle E M(r)\rangle \sim 0.35$ from $0.2-0.9 R_{500}$, with a maximum of 1.56 in the central regions and a minimum of 0.32 at $0.5 R_{500}$. The latter is somewhat smaller than that found by Neumann \& Arnaud (1999), who used a relation taken from numerical simulations to calculate $R_{500}$.

The two subsamples form distinct classes in the plot. The cool core systems, unsurprisingly, show very strong central emission and also appear to scale somewhat more tightly in the outer regions. In contrast, all of the clusters with the lowest central emission measure are classed as disturbed. 


\subsection{The $L-T$ relation}

In the left-hand panel of Fig. 2 we show the $L_{1}-T_{1}$ relation obtained with $T_{1}$ and bolometric $L_{1}$ derived from all emission interior to $R_{500}$ (i.e., equivalent to a raw, uncorrected relation). In many cases the errors are smaller than the points, a testament to the exceptional quality of the data. The best fitting power law relations derived from the BCES fits are overplotted; fits are listed in Table 2. The BCES (Y|X) slope, $2.70 \pm 0.23$, is consistent with previous determinations such as those of Markevitch (1998, $2.64 \pm 0.16)$, Arnaud \& Evrard (1999, $2.88 \pm 0.15)$, Allen \& Fabian $(1998,2.9 \pm 0.3)$ and Novicki et al. $(2002,2.82 \pm 0.32)$. The slope derived from the BCES orthogonal fit, $3.35 \pm 0.32$ is somewhat steeper, although only at slightly more than $2 \sigma$, a consequence of the very large scatter in the data. The relations of Arnaud \& Evrard (1999) and Markevitch (1998) are also plotted in the figure: their normalisations are notably lower that that found in the present work, due to their being a non-cool core cluster sample and cool core-corrected sample respectively.

The central panel of Fig. 2 shows the histogram of the log space residuals from the best fitting relations. The logarithmic scatter about the $L_{1}-T_{1}$ relation is $\sigma_{\ln L} \sim 0.7 \pm 0.1$ in both cases, and is dominated by the intrinsic component, the statistical scatter being negligible. Although the residuals present a clear skew towards higher luminosity systems, the KS probability that the residuals are drawn from a Gaussian distribution are 0.15 and 0.10 for the BCES (Y|X) and orthogonal fits, respectively. This result does not strongly exclude the Gaussian hypothesis, underlining the need for a larger sample to better understand the scatter (although note that Novicki et al. 2002 find that the log space residuals of a larger sample are consistent with a Gaussian distribution with a similar $\sigma$ to that found for the present sample).

It is interesting to investigate the factors driving the very large scatter about the $L_{1}-T_{1}$ relation. The right hand panel of Fig. 2 shows the log space deviations from the best fitting $L_{1}-T_{1}$ relations for the cooling core sample (blue stars), and for the morphologically disturbed subsample (red squares). In both cases, the subsamples clearly populate different regions of the residual space: cool core systems are preferentially located above the main relation, while morphologically disturbed systems lie below it. The best fitting power law relations to the individual subsamples are listed in Table 2. Cool core clusters have a statistically identical slope to that of the non-cool core systems, and to that of the sample as a whole. The cool core subsample has a higher normalisation than the non-cool core subsample, significant at the $>2 \sigma$ level, suggesting that the primary effect of a cooling core is to move a given system orthogonally from the standard relation. However, the logarithmic scatter about the cooling-core only relation $\left(\sigma_{\ln L}=0.48 \pm 0.13\right.$ for the BCES orthogonal fit) is higher than that about the non-cool core relation at slightly more than $1 \sigma$, reflecting the wide variety of cooling core strengths in the present sample. The logarithmic scatter about the non-cool core relation $\left(\sigma_{\ln L}=0.29 \pm 0.07\right.$, or $\left.\sigma_{\log 10} \sim 0.15\right)$, is in good agreement with that found by Arnaud \& Evrard (1999), from a sample which contained only non-cool core systems.

A similar trend is seen when the clusters are divided according to the morphology parameter. Firstly, it is clear from Fig. 2 that the disturbed clusters preferentially populate the lower envelope of the $L_{1}-T_{1}$ relation. The slopes of the relations are statistically identical for both subsamples, and in agreement with that of the entire sample, but the normalisation of the relaxed sample is higher at the $1 \sigma$ level than that of the unrelaxed sample.
This is partly due to the predominance of cool core systems in the relaxed subsample, although disturbed cool core systems do exist. The logarithmic scatter about the relations is very similar, at $\sigma_{\ln L} \sim 0.65$, although they are not well constrained.

The clear segregation of the cooling core clusters from the rest of the population (Fig. 2), together with the small relative segregation of dynamically disturbed systems and the structural similarity at large radius (Fig. 1, right hand panel), suggest that simply excluding the central region should tighten the luminosity scaling relations. Figure 3 shows the $L_{2}-T_{2}$ relation derived from emission excluding the core region, where both the luminosity and temperature are estimated in the $0.15 R_{500}<R<R_{500}$ aperture; best fitting slopes and normalisations are given in Table 2.

The BCES $(\mathrm{Y} \mid \mathrm{X})$ slope, $2.78 \pm 0.13$ is similar to the relation for that derived from all emission interior to $R_{500}$, and the slope of the BCES orthogonal fit, $2.94 \pm 0.15$ is slightly steeper but in good agreement within the $1 \sigma$ uncertainties, as is the normalisation. This relation is in excellent agreement, both in terms of slope and normalisation, with those of Markevitch (1998) and Arnaud \& Evrard (1999), which are overplotted in the same Figure.

However, the logarithmic intrinsic scatter, $\sigma_{\ln L}=0.27 \pm 0.06$ is smaller, as expected, by a factor of two. The central panel of Fig. 3 shows the histogram of the log space residuals from the best fitting BCES orthogonal relation. The overplotted curve is a Gaussian with raw $\sigma_{\ln L}=0.27$, corresponding to the scatter about the relation. The KS test probability the residuals are drawn from a Gaussian distribution is 0.54 for the BCES orthogonal fit.

The scatter is clearly reduced on exclusion of the core regions. The relative effect of the change in luminosity and temperature in the reduction of scatter can be estimated simply by comparing the values estimated in the two apertures. We find $\left\langle T_{1} / T_{2}\right\rangle=1.02 \pm 0.07$ and $\left\langle L_{1} / L_{2}\right\rangle=1.62 \pm 0.31$ for the full sample, indicating that the change in temperature is a very minor effect compared to the change in luminosity. Unsurprisingly however, the change in temperature is negative for cool core systems $\left(\left\langle T_{1} / T_{2}\right\rangle=0.96 \pm 0.07\right)$, while it is positive for non-cool core objects $\left(\left\langle T_{1} / T_{2}\right\rangle=1.05 \pm 0.05\right)$.

Table 2 also lists the fits to the different subsamples. Scatter decreases markedly (by approximately a factor of two) for the cool core subsample, as expected, but it also decreases somewhat ( $\sim 15$ per cent) for the non cool core subsample. Cool core clusters still tend to be found towards the upper envelope of the distribution, which is reflected in their slightly higher normalisation compared to non-cool core systems (although this is not significant). The slopes are stable however, and in agreement with those found for the relation derived from all emission interior to $R_{500}$. In common with the full emission sample, morphologically disturbed clusters tend to describe the lower envelope of the distribution, having a slightly lower normalisation than the full sample, although this is not significant, and a similar slope.

\subsection{The $L-Y_{\mathrm{X}}$ relation}

$Y_{\mathrm{X}}$ is an interesting quantity because simulations suggest that deviations in $M_{\mathrm{gas}, 500}$ and $T$ for a given system are anticorrelated with respect to the self-similar expectations, leading to a reduction in scatter (although thus far this is empirically untested). That $Y_{\mathrm{X}}$ is the $\mathrm{X}$-ray analogue of the integrated SZ Comptonisation parameter $Y_{\mathrm{SZ}}$ makes the calibration of its relationship with the X-ray luminosity of prime importance for the 

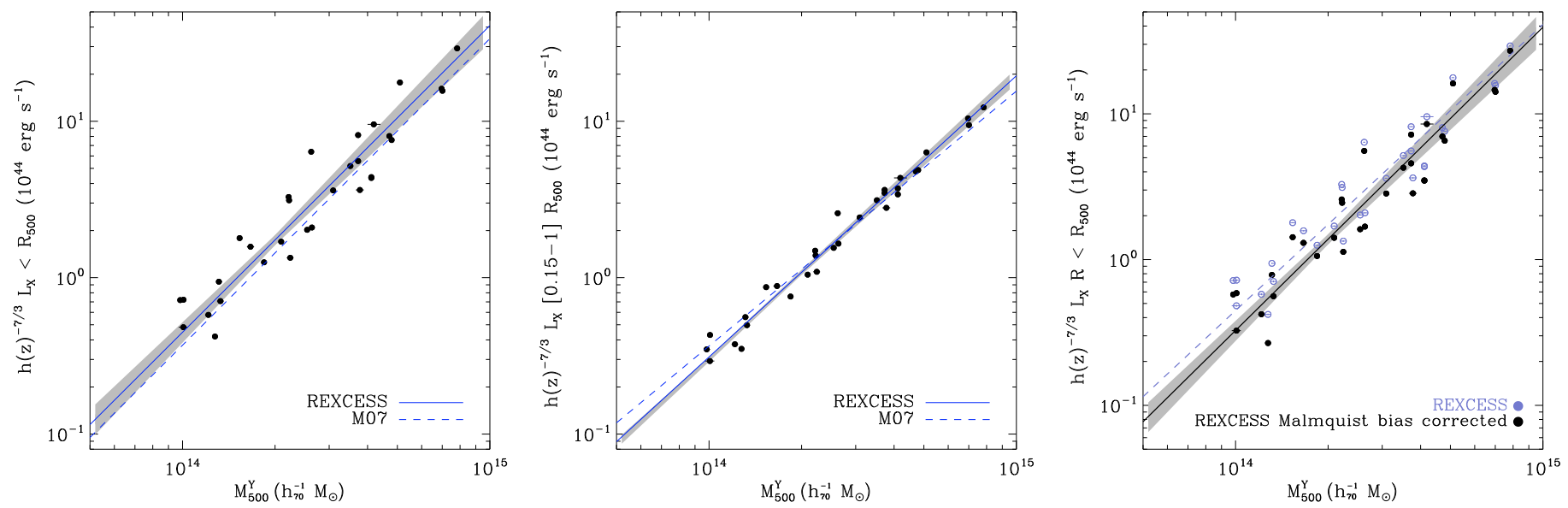

Fig. 6. $L-M_{500}$ relation for the REXCESS sample, with the mass estimated from the $Y_{\mathrm{X}}-M$ relation of Arnaud et al. (2007). Left: relation for all emission interior to $R_{500}$. Centre: relation for emission in the [0.15-1] $R_{500}$ aperture. The best fitting power law relation derived from the orthogonal BCES fit method is overplotted as a solid line. The dashed line is the fit derived by Maughan (2007) from observations of 115 galaxy clusters in the Chandra archive. Right: relation corrected for Malmquist bias as described in Appendix B.

interpretation of data from the upcoming all-sky surveys from the Planck and $e$ ROSITA satellites.

The $L_{1}-Y_{\mathrm{X}}$ relation, where the luminosity is derived from all emission interior to $R_{500}$, is shown in Fig. 4 ; the best fitting power law values are given in Table 2 . Because of the smaller scatter in these data, both BCES fitting methods give consistent results. Our relation is in good agreement with that of the Chandra archive study of 115 galaxy clusters by Maughan (2007): the slope $B=1.10 \pm 0.04$ is consistent with our BCES orthogonal value, $\alpha=1.04 \pm 0.06$, and the normalisation at $Y_{\mathrm{X}}=2 \times 10^{14} M_{\odot} \mathrm{keV}$ is only 14 per cent lower than ours. The intrinsic logarithmic scatter is $\sigma_{\ln L}=0.38 \pm 0.06$, considerably less than about the $L-T$ relation (note that since $Y_{\mathrm{X}}$ is calculated using the temperature estimated in the [0.15-1] $R_{500}$ aperture, this will tend to damp scatter somewhat). However, the residual histogram about the best fitting orthogonal BCES relation, plotted in the central panel of the same figure, has a KS probability of only 0.09 of being compatible with a Gaussian distribution.

The residual distribution for the different subsamples mirrors that of the $L_{1}-T_{1}$ relation: cool core clusters lie preferentially above and morphologically disturbed systems lie preferentially below. This fact is reflected in the different normalisations found when fitting the different subsamples: the cool core systems have the highest normalisation and the disturbed systems the lowest. However, the slope of the relation, when fitted to different subsamples, is remarkably stable at 0.96-1.06, and the slopes of all subsamples are statistically indistinguishable.

Figure 5 shows the $L_{2}-Y_{\mathrm{X}}$ relation, determined with the core emission excluded. Once again there is excellent agreement in both slope and normalisation between our relation and that of Maughan (2007). The relation is very tight: the intrinsic logarithmic scatter is only $\sigma_{\ln L}=0.16 \pm 0.04$, and the KS probability that the distribution of residuals is compatible with a Gaussian is 0.93 . For the different subsamples, Table 2 shows that the slopes are remarkably similar, ranging from 0.94 to 0.99 , and the power law normalisations for the best fitting models are segregated in a similar manner to the luminosity temperature relation, although with much reduced significance.

\subsection{The $L-M_{500}$ relation}

It is interesting to make a first examination of the slope and normalisation of the $L-M_{500}$ relation for the present sample. Since we do not have independent measures of the mass, we use the $M_{500}-Y_{\mathrm{X}}$ relation of Arnaud et al. (2007) to estimate the masses of the clusters in the sample. For the purposes of this initial investigation, we ignore the impact of the intrinsic scatter about the $M_{500}-Y_{\mathrm{X}}$ relation because it is at present not sufficiently well quantified; X-ray calibrations are necessarily available for relaxed cluster samples only, and weak lensing calibrations are at present lacking sufficient dynamic range in mass. The present approach allows us to verify the slope and normalisation of the relation under the given assumptions, to check the coherence of the slopes, and to compare with previous work using similar approaches.

The measured $L-M$ relations are summarised in Table 2 and the relations obtained for bolometric $L$ measured in both apertures are plotted in Fig. 6. The slopes of the relations, $\sim 1.8$, are consistent with the $L-T$ and $M_{500}-Y_{\mathrm{X}}$ relations, as expected. Comparing our measurements of the slope and normalisation with those of Maughan (2007), we find excellent agreement in slope for the relation derived from all emission interior to $R_{500}$, athough our normalisation is somewhat higher (by $<20$ per cent). When the core emission is excluded, the slope of Maughan's relation $(1.63 \pm 0.08)$ is somewhat shallower than our BCES orthogonal measurement $(1.80 \pm 0.05)$, at the $\sim 2 \sigma$ level. However, the normalisations are in excellent agreement.

In the right hand panel of Fig. 6 we compare the raw $L-M$ relation with that corrected for the effect of Malmquist bias. The correction procedure, and the relations for the [0.12.4] $\mathrm{keV}$ and [0.5-2] keV bands, plus comparison with the results of Vikhlinin et al. (2008), are given in Appendix B. The correction has the effect of steepening the relation slightly due to the under-representation of low-luminosity clusters on the REXCESS sample.

The scatter is, by definition, identical to that about the $L-Y_{\mathrm{X}}$ relation, and is in excellent agreement with that found by Vikhlinin et al. (2008) from a similar analysis of a larger fluxlimited sample of nearby clusters. Note that if $L \propto M^{\gamma}$, then a first order estimate of the scatter in mass is $\sigma_{\ln M} \sim \sigma_{\ln L} / \gamma$. However, this will only be true if $\sigma_{\ln L}$ is measured at fixed $M$ for a complete sample. Using the measurement of $\sigma_{\ln L}$ at fixed $T$ or $Y_{\mathrm{X}}$ introduces covariance of $T$ and $Y_{\mathrm{X}}$ into the relations, which would modify the first order scatter estimate. Nevertheless, the scale of this first order estimate of the scatter in mass is 
Table 3. Best fitting parameters for the three parameter scaling relation fits.

\begin{tabular}{lllll}
\hline \hline Relation & $C$ & $\alpha$ & $\beta$ & $\sigma_{\ln \mathrm{L}, \text { intrinsic }}$ \\
\hline$L_{1}-T_{1}-n_{e, 0}$ & $27.45 \pm 1.45$ & $2.61 \pm 0.36$ & $0.36 \pm 0.10$ & $0.47 \pm 0.04$ \\
$L_{1}-Y_{\mathrm{X}}-n_{e, 0}$ & $13.90 \pm 1.13$ & $0.99 \pm 0.04$ & $0.26 \pm 0.03$ & $0.22 \pm 0.02$ \\
$L_{1}-M-n_{e, 0}$ & $4.84 \pm 1.14$ & $1.82 \pm 0.07$ & $0.26 \pm 0.03$ & $\ldots$ \\
\hline
\end{tabular}

Data were fitted with a power law of the form $h(z)^{n} L=C\left(A / A_{0}\right)^{\alpha}\left(n_{e}\right)^{\beta}$, with $A_{0}=5 \mathrm{keV}, 2 \times 10^{14} M_{\odot} \mathrm{keV}$ and $2 \times 10^{14} M_{\odot}$, and $n=-1,-9 / 5$ and $-7 / 3$ for $T, Y_{\mathrm{X}}$ and $M$, respectively. $L_{1} / T_{1}$ : luminosity/temperature interior to $R_{500}$.

$\sigma_{\ln M} \sim 0.20-0.37$ for the full aperture and only $\sigma_{\ln M} \sim 0.09-$ 0.16 for core extracted quantities.

\subsection{Relations including a third parameter}

The presence of a cool core is clearly the factor which contributes most to the scattering of a given cluster about the best fitting relation. Figure 1 shows that the central density $n_{e, 0}$ is a very reliable indicator of cool core strength. Following O'Hara et al. (2006), it thus follows that $n_{e, 0}$ may be taken into account as a third parameter in the scaling relations.

Fitting a scaling relation of the form:

$h(z)^{n} L=C\left(A / A_{0}\right)^{\alpha}\left(n_{e}\right)^{\beta}$

where $h(z)$ is the Hubble constant normalised to its present day value and $n$ was fixed to the expected scaling with $z$, and solving for $\alpha, \beta$ and the normalisation $C$, allows us to investigate the influence of central density $n_{e, 0}$ on the scaling relations. For each relation the fit was undertaken using standard linear regression in the log-log plane. We determine the best fitting values and associated $1 \sigma$ uncertainties via 1000 bootstrap resamplings of the observed data set, and the raw scatter was estimated using the error weighted orthogonal distances to the regression line.

The resulting best fitting relations are summarised in Table 3. Dependencies on the main scaling parameter $\left(T, Y_{\mathrm{X}}\right.$ and $\left.M\right)$ are similar to those derived for the two-parameter fits to coreexcluded quantities, as expected. The scatter is comparable to that derived from a two-parameter fit to core-excluded quantities for all relations.

Thus the technique of using the central gas density appears to be a promising method for reducing scatter about the luminosity scaling relations.

\section{Discussion}

\subsection{Scatter about the relations, and correcting for it}

The REXCESS data have allowed us to investigate the sources and magnitude of the scatter about the various relations using a data set which should be representative of any X-ray selected sample of clusters.

For all emission interior to $R_{500}$, morphologically disturbed systems tend to lie below the best fitting relation to the entire whole sample; however, this is mainly due to the effect of cool core systems in the full sample, which tend to increase its normalisation. A fairer test is to compare the subsamples when the core emission is excluded: in this case, the normalisations differ in all cases by less than $1.5 \sigma$, although morphologically disturbed systems still have the lowest normalisation. In common with O'Hara et al. (2006), we find that clusters with greater morphological substructure do not exhibit more scatter about scaling relations than clusters with less substructure. This is mostly a consequence of the fact that morphologically regular systems contain a preponderance of cool core clusters. This result would also suggest that the main effect of merging is to move systems along the relation rather than orthogonal to it. In this context, we note that numerical simulations predict quasi-simultaneous boosting in temperature and luminosity at certain epochs after a merging event, which would indeed tend to move clusters along the relation.

In common with most previous investigations, we find that the vast majority of the scatter in all relations is due to the presence of cool cores, which lie systematically above the best fitting relation with an offset that appears to be related to the strength of the cool core. A fit to the cool core systems differs from a fit to the whole sample only by a normalisation factor. However, the scatter about the best fitting cool core subset relation is nearly twice that about the non cool core subset relation, reflecting the different cool core strengths.

Excluding the central emission leads to a significant reduction of the scatter about all relations. For example, the scatter about the $L-T$ relation decreases by a factor of two on exclusion of the core, and the reduction in scatter is similar for the $L-Y_{\mathrm{X}}$ relation. Correcting for the presence of a cool core by assuming a power law dependence of central density $n_{e, 0}$ with luminosity affords an alternative method to reduce scatter. The reduction in scatter obtained by the use of $n_{e, 0}$ is of the same order as that obtained from simple core exclusion. We note that core exclusion may be difficult in the case of distant clusters or those detected with very low signal to noise, and in these circumstances it may be preferable to use the central density or surface brightness to reduce scatter about the scaling relations.

\subsection{Slope of the relations}

The X-ray luminosity of a cluster can be written (Arnaud \& Evrard 1999):

$L(T)=f_{\mathrm{gas}}^{2}(T)[M(T) \Lambda(T)] \hat{Q}(T)$

where $\Lambda(T)$ is the cooling function. $\hat{Q}(T)$, introduced by Arnaud $\&$ Evrard (1999), is equal to $\left\langle\rho_{\text {gas }}^{2}\right\rangle /\left\langle\rho_{\text {gas }}\right\rangle^{2}$, with the angle brackets denoting an intrinsic volume average. $\hat{Q}(T)$ is thus a dimensionless structure factor which depends only on the spatial distribution of the gas density (e.g., clumpiness at small scale, shape at large scale, etc). With the set of additional assumptions (i) pure bremsstrahlung emission $\left[\Lambda(T) \propto T^{1 / 2}\right]$; (ii) virial equilibrium $\left[M \propto T^{3 / 2}\right]$; identical internal cluster structure $\left[\hat{Q}(T)=C_{1}\right]$; constant gas mass fraction $\left[f_{\text {gas }}(T)=C_{2}\right]$, we arrive at the standard self-similar expectation for the luminosity-temperature relation, $L \propto T^{2}$. The self-similar $L-Y_{\mathrm{X}}$ relation can be obtained from combination of the gas mass-luminosity and luminositytemperature relations to give $L \propto Y_{\mathrm{X}}^{4 / 5}$. Combining the selfsimilar mass-temperature and luminosity-temperature relations leads to a dependence of luminosity with mass of $L \propto M^{4 / 3}$.

In common with most previous work on the subject, we find that the slope of the $L-T$ relation of the REXCESS sample is steeper than the prediction from the expectations of self-similar collapse models. The steeper slope is found consistently in all subsamples, and in all cases the statistical precision of the data allow us to rule out the self-similar predictions. We find similar results for the $L-Y_{\mathrm{X}}$ relation, where the observed slope of $\sim 1.0$ is significantly steeper than the self-similar expectation of 0.8 , and for the $L-M_{500}$ relation, where the observed slope of 1.8 is steeper than the expected value of 1.3 . These facts imply that 

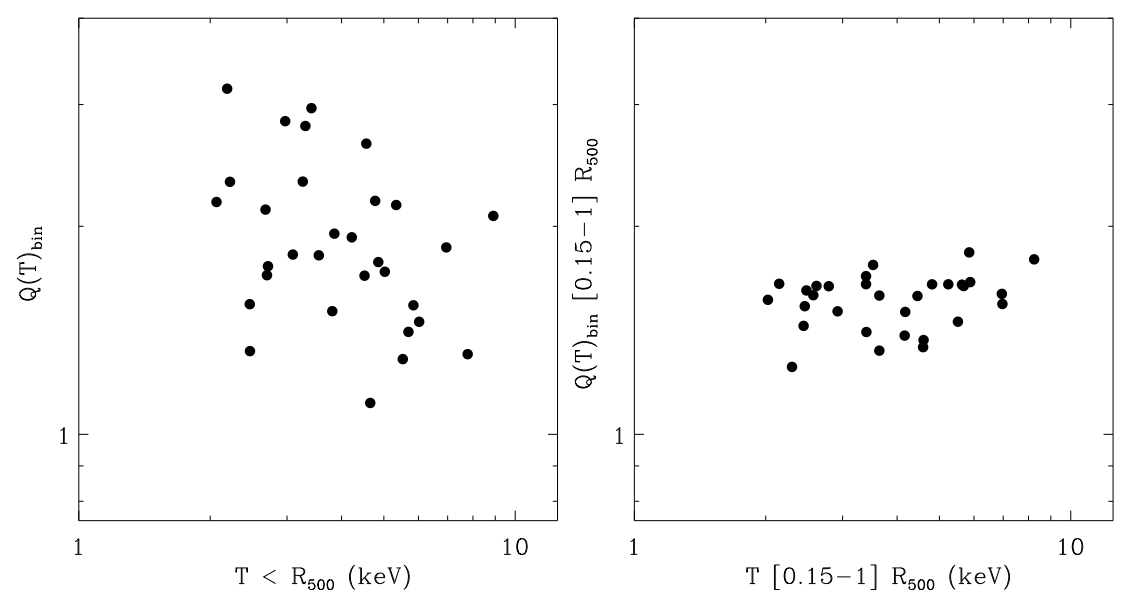

Fig. 7. Structure factor $\hat{Q}(T)_{\text {bin }}=$ $\left\langle\rho_{\text {gas }}^{2}\right\rangle /\left\langle\rho_{\text {gas }}\right\rangle^{2}$, estimated from the gas density profiles, versus temperature. Left: quantities estimated from all emission interior to $R_{500}$. Right: quantities estimated in the $[0.15-1] R_{500}$ aperture. There is no significant dependence of $\hat{Q}(T)_{\text {|bin }}$ on temperature on a Kendall's $\tau$ test in either case.

one or more of the assumptions listed above does not hold for the real cluster population.

The assumption of pure bremsstrahlung is not strictly valid since line emission becomes increasingly important as the temperature decreases, having the effect of flattening the relation as lower temperature systems are boosted in luminosity. While the lower temperature limit of the REXCESS sample, $2 \mathrm{keV}$, should suffice to minimise these effects, systematic differences in the metallicity between objects may serve to change the temperature dependence of the X-ray emission from the expected value of $T^{1 / 2}$. We tested this using the measured temperatures and abundances of the REXCESS sample, finding a best fitting power law relation of 0.5 , in full agreement with the expected dependence.

The assumption of virial equilibrium leads to the expected relation $M \propto T^{3 / 2}$ between total mass and temperature. A topic of vigorous debate in previous years, several recent investigations of the $\mathrm{X}$-ray mass-temperature relation have shown percent-level agreement in normalisation and that the slope is not greatly different from the self-similar expectation (Arnaud et al. 2005; Vikhlinin et al. 2006), although it may be slightly steeper (Arnaud et al. 2005; Sun et al. 2008).

The question of structural regularity has also received quantitative tests in recent years. For instance, there is now converging evidence that the total mass density profiles of galaxy clusters and groups scale quasi-self-similarly with a mass dependence that is in good agreement with predictions from numerical simulations (eg., Pointecouteau et al. 2005; Vikhlinin et al. 2006; Gastaldello et al. 2007). On the mass scales we are considering here, the variation of the total mass density concentration with mass is in fact consistent with zero (Pointecouteau et al. 2005; Vikhlinin et al. 2006). This would imply that a variation of cluster dark matter structure with mass cannot be responsible for the steepening of the $L-T$ relation, at least in the mass range covered by the present data.

However, the baryonic components of clusters are subject to somewhat different physics, and there are indications that the large scale ICM density structure is temperature/mass dependent. The clear correlation of the slope of the gas density profile measured in the radial range [0.3-0.8] $R_{500}$ with temperature seen in the REXCESS analysis of Croston et al. (2008) is one example. At the same time Croston et al. have shown that the temperature dependence of the relative dispersion of scaled gas density profiles has all but disappeared at $0.7 R_{500}$, suggesting that clusters become increasingly structurally similar at larger radii. This is borne out in the present data when we examine the more powerfully diagnostic structure factor $\hat{Q}(T)_{\mid \text {bin }}=\left\langle\rho_{\text {gas }}^{2}\right\rangle /\left\langle\rho_{\text {gas }}\right\rangle^{2}$, where the average is taken over the radial gas density profile.
This quantity is identical to that presented in Arnaud \& Evrard (1999) except that we use a fully deconvolved, deprojected gas density profiles rather than $\beta$-model fits. $\hat{Q}(T)_{\mid \text {bin }}$ effectively probes the variation of the large scale shape of the gas density with temperature, that is to say, variation of the gas concentration with mass. However, since $\rho_{\text {gas }}(r)$ is derived from spherically symmetric deprojection of the surface brightness profile, by construction $\rho_{\text {gas }}(r)$ is $\sqrt{\left\langle\rho_{\text {gas }}^{2}\right\rangle}$, where $\left\langle\rho_{\text {gas }}^{2}\right\rangle$ is the average within each radial shell. Thus we emphasise that $\hat{Q}(T)_{\mid \text {bin }}$ is only a partial estimator of $\hat{Q}(T)$ which does not probe more subtle effects such as variations of the gas clumpiness with mass, or substructuring at small scale.

In Fig. 7 we plot $\hat{Q}(T)_{\mid \text {bin }}$ versus system temperature for both apertures considered in the present work. The evidence for a correlation between $\hat{Q}(T)_{\mid b i n}$ and system temperature is very weak on a Kendall's $\tau$ test, being significant at only $\sim 8$ per cent for the full aperture and $\sim 15$ per cent for the $[0.15-1] R_{500}$ aperture. Interestingly, the standard deviation of $\hat{Q}(T)_{\mid \text {bin }}$ in the [0.151] $R_{500}$ aperture, $\sigma_{\hat{Q}(T)}=0.14$, gives an observational limit of the variation of cluster structure outside the core regions at fixed temperature. That it is only on the order of 15 per cent argues for a cluster population of remarkable structural similarity. It is thus unlikely that a systematic dependence of cluster structure on temperature/mass can be a major cause of steepening of the $L-T$ relation of the present sample. However, the effect of a systematic dependence of gas structure at smaller scale with temperature/mass remains an open issue. More detailed assessment of the gas clumpiness requires combining X-ray data with high quality SZ data.

A more likely explanation is if the gas mass-temperature relation $M_{\mathrm{gas}}-T$ deviates from predictions (or equivalently, if the gas mass fraction, $f_{\mathrm{gas}}=M_{\mathrm{g}, 500} / M_{500}$ varies with mass), as was discussed in previous work based on ROSAT data (e.g., Neumann \& Arnaud 2001; Mohr et al. 1999). Recent results on the relaxed cluster sample of Arnaud et al. (2007) and on the REXCESS sample itself (Croston et al. 2008) have shown that $M_{\mathrm{g}, 500} \stackrel{\propto}{\sim} T^{2}$, in agreement with previous work, thus implying a steeper dependence than the self-similar prediction of $M_{\text {gas }} \propto T^{3 / 2}$. Such a dependence of $M_{\text {gas }}$ on $T$ would imply $f_{\text {gas }} \propto M^{1 / 3}$ for a self-similar $M \propto T^{3 / 2}$ relation.

The left hand panel of Fig. 8 shows the $f_{\text {gas, } 500}-M_{500}$ relation for 41 systems ranging from $10^{13}$ to $10^{15} M_{\odot}$ for which gas mass fraction estimates derived from hydrostatic mass measurements are available (Arnaud et al. 2007; Vikhlinin et al. 2006; Sun et al. 2008). The trend of mean gas fraction with mass is evident to the 

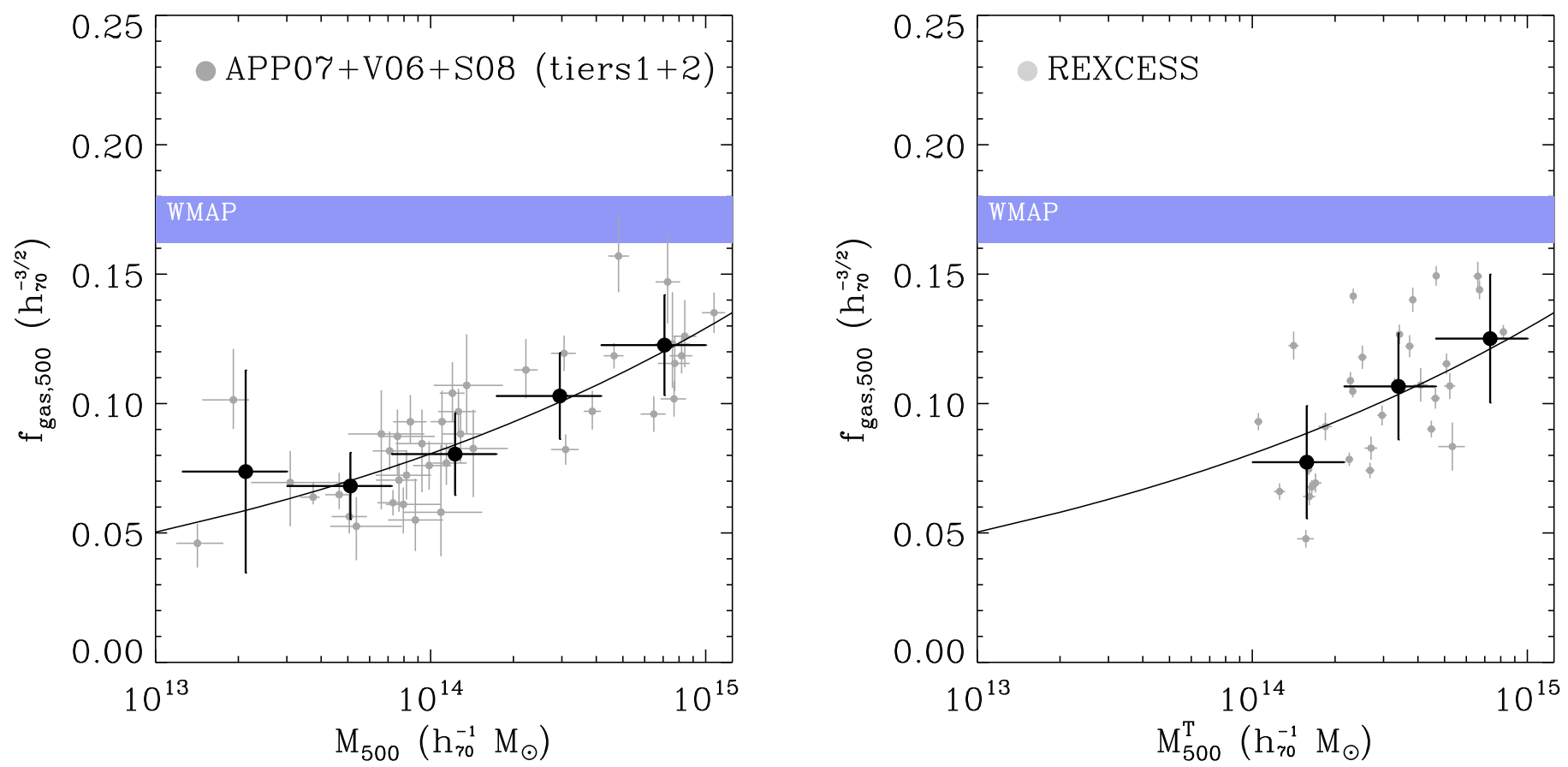

Fig. 8. Gas mass fraction vs mass. Left panel: trend of gas mass fraction versus mass derived from X-ray measurements of 41 groups and clusters with high quality hydrostatic mass estimates (Arnaud et al. 2007; Vikhlinin et al. 2006; Sun et al. 2008). Grey points are actual measurements; black points are mean values in logarithmic mass bins. The solid line is the orthogonal BCES fit to the unbinned data, $f_{\text {gas,500 }} \propto M^{0.2}$. Right panel: approximate gas mass fraction versus mass measurements for the REXCESS sample, where the masses have been estimated from the $M_{500}-T$ relation of Arnaud et al. (2005). Black points again show the mean trend for three logarithmic mass bins. The solid line is the same as in the left panel. The band illustrates the WMAP 5-Year baryon fraction constraints (Dunkley et al. 2009).

eye. To better illustrate this, we have divided the data into five approximately equal logarithmic total mass bins and calculated the mean and standard deviation of the gas mass fraction measurements in each bin. A BCES orthogonal fit to the combined (not binned) data set in log-log space yields the relation:

$$
\begin{aligned}
h(z)^{3 / 2} \ln f_{\text {gas }, 500}= & (-2.37 \pm 0.03) \\
& +(0.21 \pm 0.03) \times \ln \left(M / 2 \times 10^{14} M_{\odot}\right)
\end{aligned}
$$

with $\sigma_{\operatorname{lnf}}=0.12 \pm 0.03$ dispersion. The right hand panel of Fig. 8 shows the corresponding trend of $f_{\text {gas }, 500}$ with cluster mass for the REXCESS sample, where the data values have been estimated using the $M-T$ relation of Arnaud et al. (2005). Dividing the data into three mass bins and averaging the gas mass fraction measurements in each bin yields the thick solid points, which are in good agreement with the best fitting relation to the combined data from hydrostatic estimates discussed above.

The trend in $f_{\text {gas }}$ implies a decrease in gas content in poorer systems relative to higher mass systems, a fact which manifests itself in an increase in ICM entropy and consequent suppression in luminosity at lower masses. The two most likely physical possibilities are a variation with mass in the efficiency of conversion of baryons into stars or in situ non-gravitational energy input from e.g., supernova feedback or AGN (e.g. Puchwein et al. 2008; Bower, et al. 2008), or a combination of the two. In a forthcoming paper we will use the entropy distributions to probe the source(s) and extent of the entropy redistribution.

Better constraints in the group regime are still required, especially in the light of the increased scatter apparently seen there (Osmond \& Ponman 2004; but see Sun et al. 2008).

\section{Conclusions}

We have presented a detailed study of the luminosity scaling relations of REXCESS, a galaxy cluster sample selected by X-ray luminosity in such a way as to optimally sample the cluster X-ray luminosity function. REXCESS contains objects of different dynamical states with a range of core X-ray properties, allowing us to investigate the effect of the presence of these systems on the scaling relations. The homogeneous nature of the sample data, which have all been observed with the same satellite to approximately the same depth, combined with an analysis approach based on extraction of relevant quantities within scaled apertures, has been designed to minimise measurement scatter. We found the following results:

- The slope of the luminosity-temperature, luminosity- $Y_{\mathrm{X}}$ and luminosity-mass relations are all steeper at greater than 99 per cent confidence, than expected for self-similar gravitational collapse scenarios.

- The dependence of the radially averaged structure factor $\hat{Q}(T)_{\mid \text {bin }}$ on temperature, where both quantities have been estimated for the first time within $R_{500}$, is not significant either when measured from all emission, or from core-excluded emission. This suggests that, contrary to previous results, structural variation cannot be a significant contributor to the steepening of the relations unless there is a very strong temperature/mass dependence of gas clumpiness. Furthermore, the scatter in $\hat{Q}(T)_{\mid b i n}$ measured in the [0.15-1] $R_{500}$ aperture is $\sim 15$ per cent, illustrating the remarkable structural similarity of the present sample in the outer regions.

- There is strong evidence for a decrease in gas mass content in poorer systems relative to higher mass systems, or in other words, a dependence of the gas mass fraction on total mass. This effect is clearly seen in the $M_{\mathrm{gas}}-T$ 
relation of the present sample (Croston et al. 2008) and has been seen in many previous samples. Using the total mass determined from published hydrostatic estimates of a combined sample of clusters and groups spanning $10^{13}-10^{15} M_{\odot}$, we find that the gas mass fraction depends on total mass such that $f_{\text {gas }} \propto M^{0.21 \pm 0.03}$. The trend in the REXCESS data, when total masses are estimated using the $M-T$ scaling relation, is similar. This dependence is the dominant cause of the steepening of the X-ray luminosity scaling relations.

- For the whole sample, the scatter of the X-ray luminositytemperature relation derived from all emission interior to $R_{500}$ is up to 75 per cent depending on the exact fitting method; the scatter is less than 40 per cent for the X-ray luminosity- $Y_{\mathrm{X}}$ relation. The scatter is not strongly compatible with a Gaussian distribution in either case, the distribution being characterised by a tail caused by the presence of cool core systems.

- Cooling core systems, the one-third of the sample with the highest central density, describe the high luminosity envelope and contribute the majority of the variance to the relations. A fit only to the cool core systems suggests that they are offset from the relation by a simple normalisation factor. However, there is nearly two times more logarithmic scatter about the X-ray luminosity-temperature relation for cool core systems compared to that for non cool core systems. This suggests that there are large differences in the core structure even for cool core systems as a class.

- Systems exhibiting morphological substructure tend towards the lower luminosity envelope of the relations. Partly this is due to the increase in normalisation of the total sample due to the presence of cool core systems. The scatter about the X-ray luminosity-temperature relation of morphologically disturbed systems is 65 per cent, compared to a scatter of 48 per cent for cool core systems; within the uncertainties, the variance is in fact identical. Furthermore, the scatter in morphologically disturbed systems is identical to that for morphologically relaxed systems, due partly to the preponderance of cool core systems in the relaxed subsample.

- Simple exclusion of the emission interior to $0.15 R_{500}$ results in a reduction of scatter in all relations. For the X-ray luminosity- temperature relation, the natural logarithmic scatter is 30 per cent, a reduction of more than a factor of two. Similarly significant reductions are seen in other relations. After exclusion of the core, the scatter in luminositytemperature and luminosity- $Y_{\mathrm{X}}$ relations is well described with a Gaussian distribution at $>85$ per cent confidence. A reduction in scatter can also be achieved by considering the central gas density, $n_{e, 0}$, as a third parameter in the scaling relations. The $L-T-n_{e, 0}$ relation has a natural logarithmic scatter of 47 per cent; the $L-Y_{\mathrm{X}}-n_{e, 0}$ relation has a scatter of 22 per cent.

- Using $Y_{\mathrm{X}}$ as a mass proxy, a Malmquist bias corrected luminosity mass relation for REXCESS is steeper than the raw relation due to the under-representation, for a given mass, of low luminosity clusters in the sample.

The behaviour of the observed luminosity scaling relations thus appears to be driven principally by a mass dependence of the total gas content. Plausible physical explanations for the dependence are a variation with mass in the efficiency of conversion of baryons into stars or in situ heating after accretion. Greater understanding of the source of the dependence will require deep observations of a similarly representative sample of group scale haloes, to measure accurate luminosities and probe the underlying physical causes; furthermore, precise calibration of the evolution of the scaling relations is needed, ideally with a similarly-selected distant cluster sample, to probe the effect over time.

Acknowledgements. We thank the referee for a useful report which helped to improve the quality of the paper. During the course of this work, we had fruitful discussions with J. Ballet, A. E. Evrard, D. Pierini, E. Pointecouteau, T. J. Ponman and A. Vikhlinin. Pertinent comments on the manuscript were also received from S. Borgani, J. P. Henry, R. Kneissl, T. H. Reiprich, A. J. R. Sanderson, and G. M. Voit. G.W.P. acknowledges support from DfG Transregio Programme TR33. The present work is based on observations obtained with XMM-Newton, an ESA science mission with instruments and contributions directly funded by ESA Member States and the USA (NASA). The XMM-Newton project is supported in Germany by the Bundesministerium für Wirtschaft und Technologie/Deutsches Zentrum für Luft- und Raumfahrt (BMWI/DLR, FKZ 50 OX 0001), the MaxPlanck Society and the Heidenhain-Stiftung.

\section{Appendix A: Cluster image gallery, sorted by cool core and $\langle\mathbf{w}\rangle$ classification}

As an aid to visualisation of the sample, in this Appendix we present the images of each cluster that were used to calculate the centroid shift parameter $\langle w\rangle$. We remind the reader that $\langle w\rangle$ was evaluated with the central $0.1 R_{500}$ excised, to avoid biasing as a result of the highly peaked surface brightness of cool core systems. The images are derived from the three EPIC detectors and have been corrected for vignetting; in addition, point sources have been removed and replaced by Poisson noise sampled from counts in an annulus surrounding the excised source.

Figures A.1 and A.2 show the resulting images. Contours increase in steps of $\sqrt{2}$. As in the original REXCESS paper (Böhringer et al. 2007), the colour table of each panel is scaled by a factor of $L[0.1-2.4]^{0.22}$, derived from the theoretical relationships between surface brightness and radius $\left(S_{\mathrm{X}} \propto R\right)$, radius and mass $\left(R \propto M^{1 / 3}\right)$ and luminosity and mass $\left(L \propto M^{4 / 3}\right)$.

Clusters are divided into cool core and non cool core subsamples and then arranged in order of increasing centroid shift parameter $\langle w\rangle$. Cool core systems generally appear more morphologically undisturbed; however, the increase in morphological complexity is always evident at higher values of $\langle w\rangle$. Note that two clusters are classified as both cool core and morphologically disturbed: RXC J1302 +0230 and RXC J2319 -7313. Figure A.1 shows that they exhibit both centrally peaked surface brightness and morphological complexity, as expected.

\section{Appendix B: Survey luminosities and associated scaling relations}

\section{B.1. Introduction}

Past (e.g., ROSAT) and future (e.g., eROSITA) X-ray survey satellites are primarily sensitive in the soft X-ray band. In this Appendix we give the soft band luminosities of the REXCESS sample, together with the scaling relations between luminosity and temperature and $Y_{\mathrm{X}}$. All quantities are calculated as described in Sect. 2.2.

\section{B.2. Correction for selection bias}

Selection effects can bias the observed scaling relations if not all clusters are completely sampled in a well defined test volume. The classical problem is the selection bias of an X-ray flux-limited sample, where the more luminous clusters are sampled from a larger volume than the less luminous clusters. Thus for any cluster property that is correlated with luminosity and 

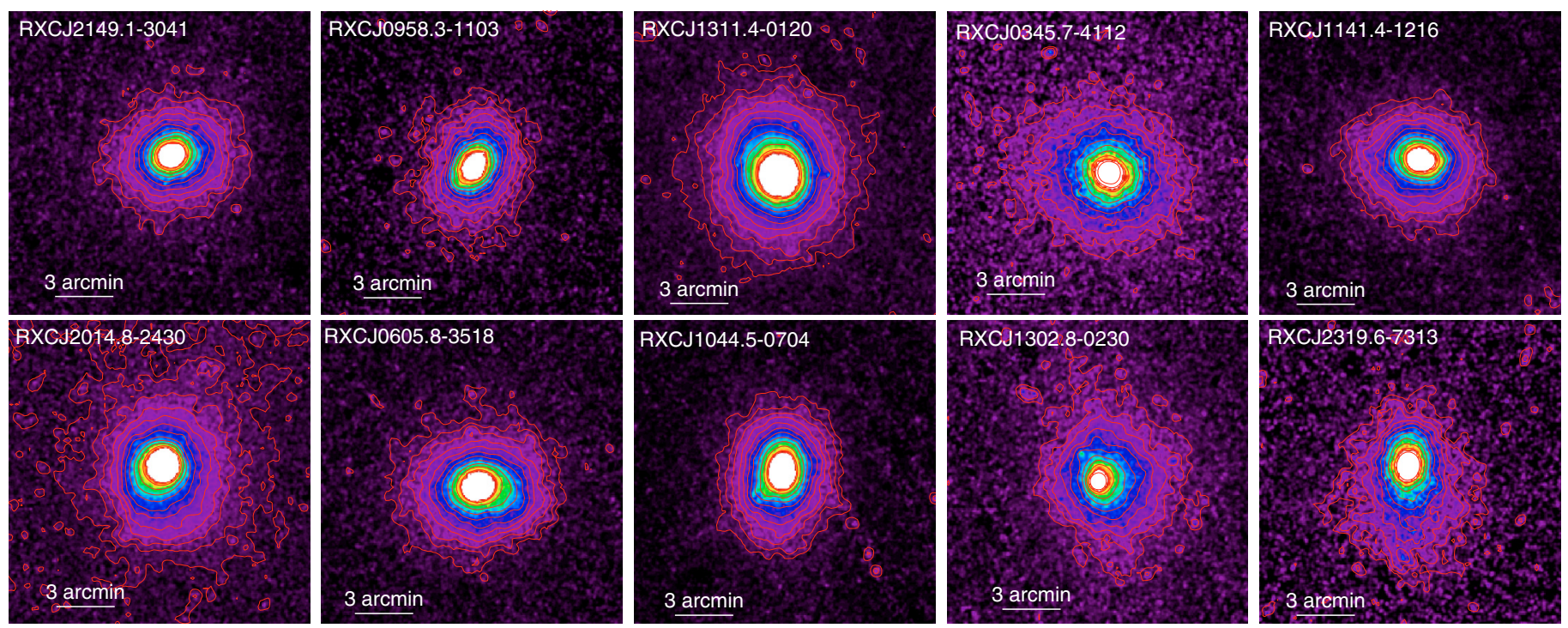

Fig. A.1. Cool core clusters, sorted from top left to bottom right in order of increasing centroid shift parameter, $\langle w\rangle$. The images are derived from the three EPIC detectors and have been corrected for vignetting. Point sources have been removed and replaced by Poisson noise sampled from counts in a surrounding annulus. Contours increase in steps of $\sqrt{2}$. The colour table of each panel is scaled by a factor of $L_{\mathrm{X}}^{0.22}$ (see Böhringer et al. 2007, for details). Note that RXC J1302+0230 and RXC J2319 -7313 are classified as both cool core and morphologically disturbed.

features a finite scatter, the more luminous clusters will always be over-represented with respect to the less luminous clusters. This effect has previously been accounted for in flux-limited surveys in works by e.g., Ikebe et al. (2002); Stanek et al. (2006); Pacaud et al. (2007); Vikhlinin et al. (2008). In the case of REXCESS, the survey geometry is more complex, and has properties of both a flux- and a volume-limited sample.

The basis for the calculation of the bias effect is the survey selection function, which for our case specifies the size of the sampling volume as a function of X-ray luminosity. The selection function is given by three ingredients (i) the sky area covered by REFLEX ( $\sim 4.24 \mathrm{st})$ and the luminosity intervals and redshift shells defined for the REXCESS sample selection; (ii) the incompleteness of the sky coverage due to low exposure regions of the ROSAT All-Sky Survey (RASS); and (iii) the flux measurement error which introduces a dispersion between the observed and true X-ray luminosity. For the luminosity and redshift boundaries we use the definitions given in Table 1 of Böhringer et al. (2007). For those bins containing more clusters than the four clusters per bin selected for REXCESS we use an appropriate fractional weighting factor. The incompleteness is given by the sensitivity function in Table 8 of Böhringer et al. (2004), illustrated in Fig. 23 of Böhringer et al. (2001), which accounts for the fact that not the complete redshift shell given in step (i) is sampled, but there is an incomplete coverage due to lack of sensitivity in some areas with low exposure and/or high interstellar column density, $N_{\mathrm{H}}$. An additional factor must be used in connection with the sensitivity function since REXCESS clusters were selected to have at least 30 counts in the RASS; this is taken into account as explained in Böhringer et al. (2001, 2004). For the first seven bins we used a further restriction in the REXCESS selection, $N_{\mathrm{H}} \leq 6 \times 10^{20} \mathrm{~cm}^{-2}$, so for these bins we have recalculated the sky coverage for the restricted sky region meeting the $N_{\mathrm{H}}$ criterion. This and some other corrections are small in our case (e.g., the part of the sky covered up to the nominal fluxlimit for a minimal detection of 30 photons increases from $78 \%$ to $79.5 \%$ for the low $N_{\mathrm{H}}$ region), and will not significantly affect the final results here, but it is nevertheless important to have all these effects under control.
The resulting selection volume function shown in the left hand panel of Fig. B.1 is a step function for the 9 luminosity bins of REXCESS, with the steps slightly tilted due to correction (ii). Folding in the scatter between measured and intrinsic luminosity, which is assumed to be $\sim 10 \%$, smooths the step function. It is monotonically increasing with X-ray luminosity. It implies that luminous clusters are more represented than less luminous systems within REXCESS. This can then be straightforwardly included in a weighting factor for the clusters of different luminosity for a given mass. One approach is to give each cluster a weighting factor inverse to the selection volume ratio of the measured and nominal luminosity for given mass before fitting a scaling relation. Or, as we do here, we can follow a recipe analogous to that described by Vikhlinin et al. (2008, Apendix A.2) where the mean bias for given mass is calculated via,

$$
\begin{aligned}
\text { Bias }\left(\ln L \mid \ln L_{0}\right) & =\langle\ln L\rangle-\ln L_{0} \\
& =\frac{\int_{-\infty}^{+\infty} \Delta \ln L p\left(\ln L \mid \ln L_{0}\right) V_{\mathrm{sel}}(\ln L) d \ln L}{\int_{-\infty}^{+\infty} p\left(\ln L \mid \ln L_{0}\right) V_{\mathrm{sel}}(\ln L) d \ln L}
\end{aligned}
$$

where $L_{0}$ is the mean (zero scatter) $L$ for given mass, $\Delta \ln L=$ $\ln L-\ln L_{0}$, and $p\left(\ln L \mid \ln L_{0}\right)$ characterizes the scatter of $L$ in the mass - luminosity relation, assumed to be lognormally distributed, and given by the observed scatter in the $L-M_{Y}$ relation (Table 2). The bias factor is shown as a function of luminosity in the right hand panel of Fig. B.1.

A final subtlety is that the luminosities used as the basis of the REXCESS selection, and thus for the bias calculation above, are those calculated as in the original REFLEX catalogue. These were iteratively calculated in the $[0.1-2.4] \mathrm{keV}$ band in the detection aperture and extrapolated to an assumed radius of $R_{200}$, and are thus not equivalent to the luminosities derived in this paper (see Böhringer et al. 2004, for luminosity calculation details; the appropriate REFLEX luminosities for the REXCESS sample are given in Table 3 of Böhringer et al. 2007). We fit a linear relation in $\log -\log$ space between the present luminosities and those from REFLEX, finding $L_{\text {REFLEX }}=1.15 \times$ $\left([0.1-2.4] \mathrm{keV} L_{\text {REXCESS }}\right)^{0.94}$. The bias correction factor for the 

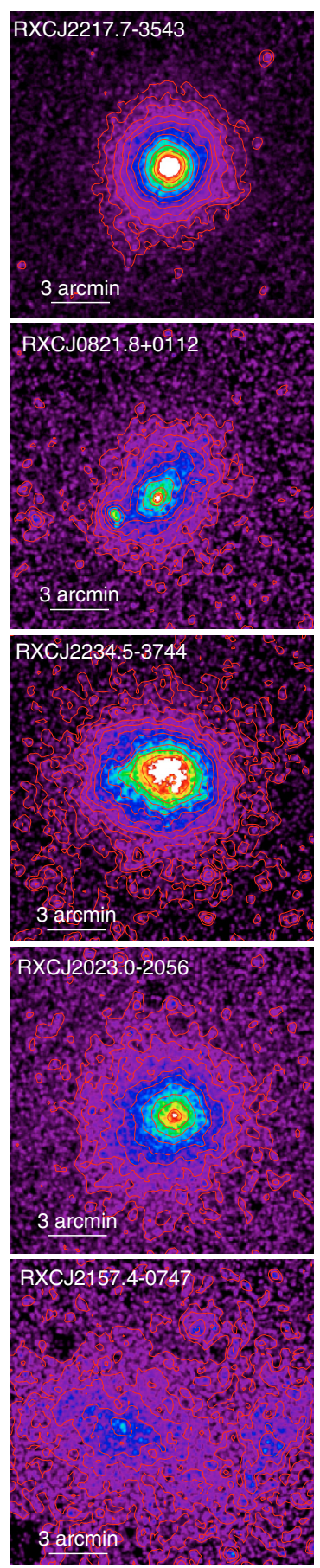
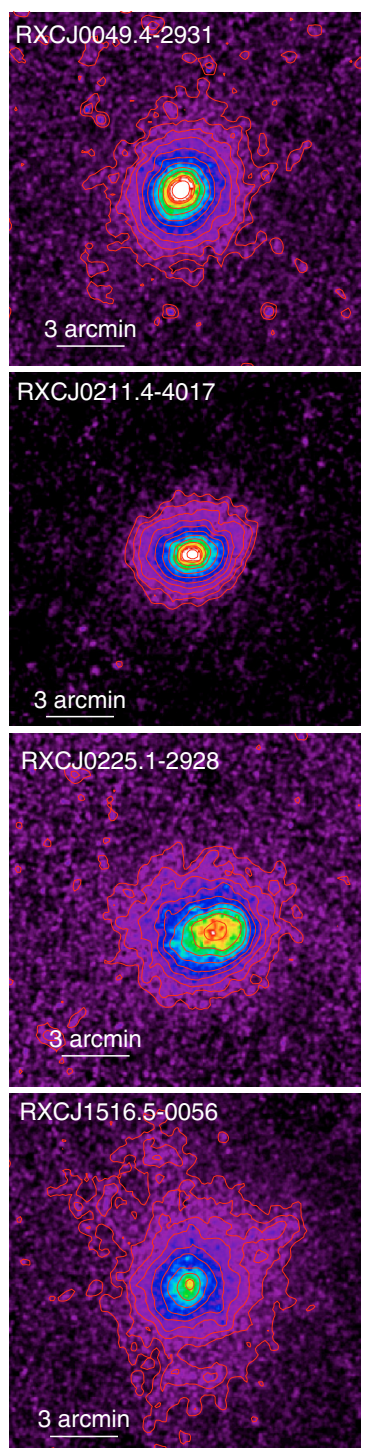
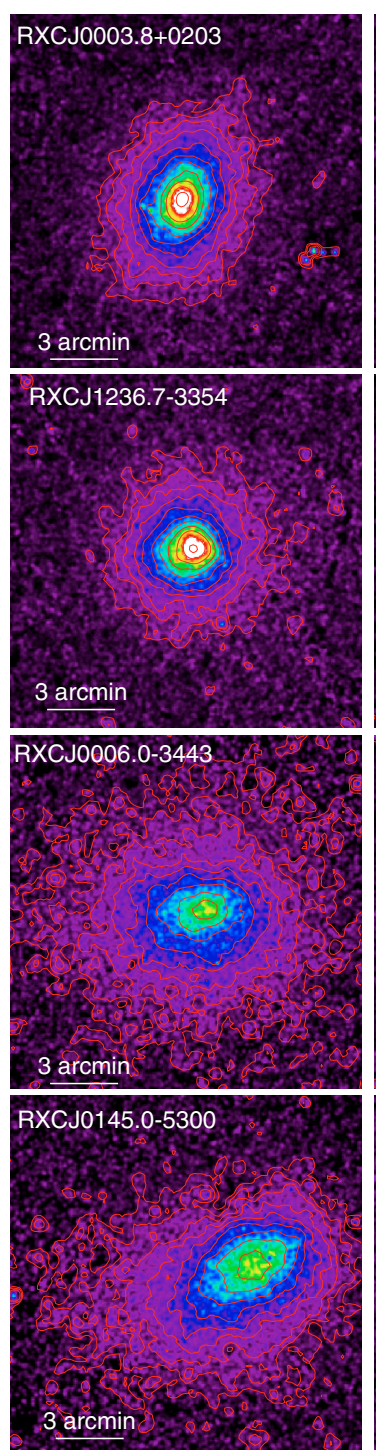
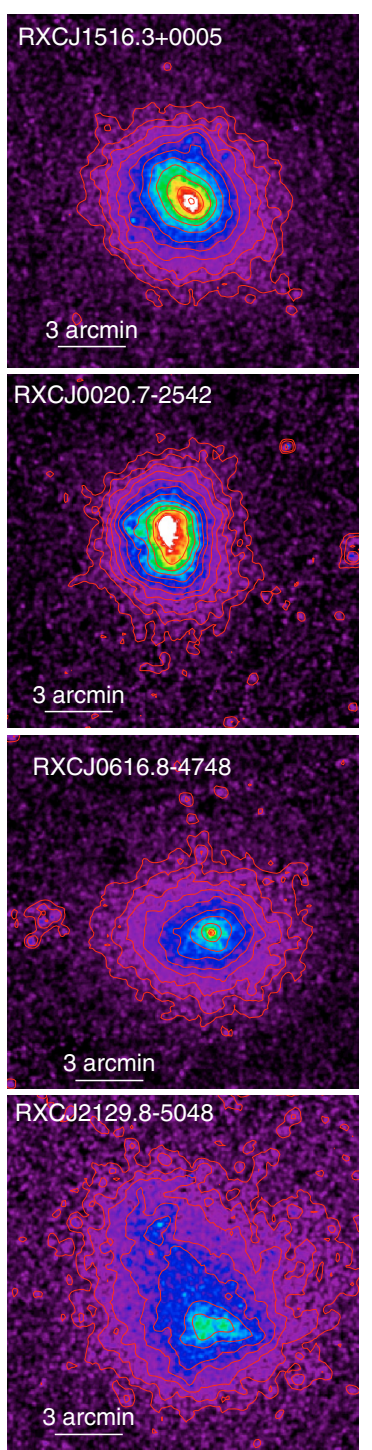
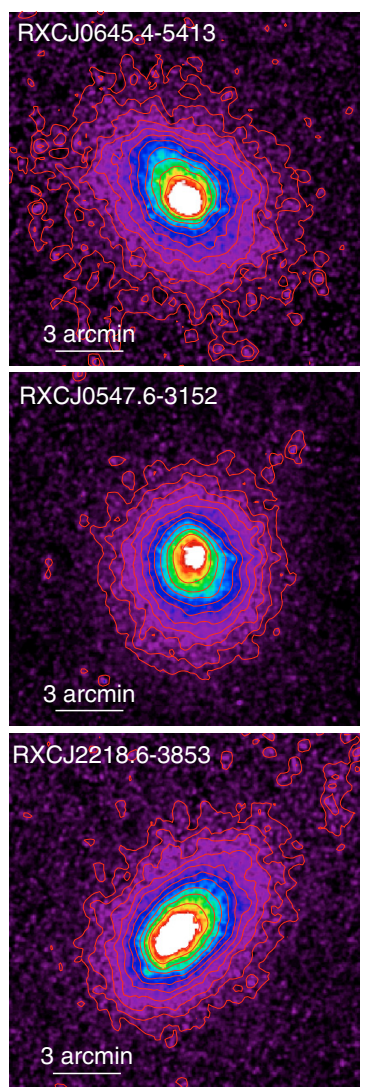

RXCJ2048.1-1750

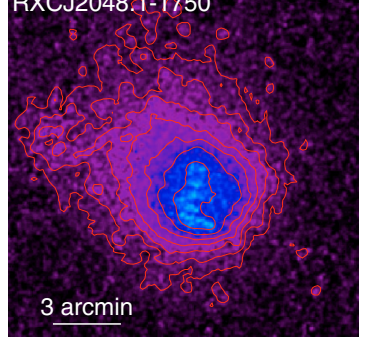

Fig. A.2. Non-cool core clusters, sorted from top left to bottom right in order of increasing centroid shift parameter, $\langle w\rangle$.

appropriate luminosity, Bias $\left(\ln L \mid \ln L_{0}\right)$, is then applied to each data point in the sample and the relation is refitted.

The Malmquist bias-corrected bolometric $L-M_{Y}$ relation is shown in the right hand panel of Fig. 6, and the corresponding fitted power law relation is given in Table 2. The correction steepens the relation somewhat, due to the under-representation, at a given mass, of low luminosity clusters in the REXCESS sample.

\section{B.3. Comparison to other results}

The corrected relations for the two survey bands are also given in Table B.2. The left hand panel of Fig. B.2 shows the raw and corrected [0.1-2.4] keV band relation compared to previous determinations from an X-ray hydrostatic analysis assuming isothermality (Reiprich \& Böhringer 2002) and a stacked weak lensing analysis (Rykoff et al. 2008). We convert their $L-M_{200}$ relations to $M_{500}$ using a standard NFW model with a concentration parameter of $c_{500}=3.2$, the average concentration derived from the total mass profiles of the morphologically regular cluster sample discussed in Pointecouteau et al. (2005). Our corrected relation has a 25 per cent higher normalisation than that of Rykoff et al. at our fiducial pivot point of $2 \times 10^{14} M_{\odot}$. The Reiprich \& Böhringer relation has a 6 per cent lower normalisation than our corrected relation at the same mass scale. 
Table B.1. Cluster properties in the soft X-ray band suitable for surveys. Luminosities are given in the [0.1-2.4] keV band appropriate for ROSAT, and also the [0.5-2] keV band. All quantities are calculated assuming $\Omega_{M}=0.3, \Omega_{\Lambda}=0.7$, and $h_{0}=0.7$.

\begin{tabular}{|c|c|c|c|c|c|c|c|}
\hline $\begin{array}{c}\text { Cluster } \\
(1)\end{array}$ & $\begin{array}{c}z \\
(2) \\
\end{array}$ & $\begin{array}{l}T_{1} \\
(3) \\
\end{array}$ & $\begin{array}{c}L[0.1-2.4]_{1} \\
(4)\end{array}$ & $\begin{array}{c}L[0.5-2]_{1} \\
(5)\end{array}$ & $\begin{array}{l}T_{2} \\
(6) \\
\end{array}$ & $\begin{array}{c}L[0.1-2.4]_{2} \\
(7)\end{array}$ & $\begin{array}{c}L[0.5-2]_{2} \\
(8)\end{array}$ \\
\hline RXC J0003+0203 & 0.0924 & $3.85_{-0.09}^{+0.09}$ & $1.02_{-0.01}^{+0.01}$ & $0.63_{-0.00}^{+0.00}$ & $3.64_{-0.09}^{+0.09}$ & $0.65_{-0.01}^{+0.01}$ & $0.40_{-0.00}^{+0.00}$ \\
\hline RXC J0006-3443 & 0.1147 & $5.03_{-0.19}^{+0.19}$ & $1.96_{-0.02}^{+0.02}$ & $1.21_{-0.01}^{+0.01}$ & $4.60_{-0.16}^{+0.21}$ & $1.58_{-0.02}^{+0.02}$ & $0.97_{-0.01}^{+0.01}$ \\
\hline RXC J0020-2542 & 0.1410 & $5.69_{-0.11}^{+0.11}$ & $2.93_{-0.02}^{+0.02}$ & $1.81_{-0.01}^{+0.01}$ & $5.24_{-0.15}^{+0.15}$ & $1.92_{-0.02}^{+0.02}$ & $1.18_{-0.01}^{+0.01}$ \\
\hline RXC J0049-2931 & 0.1084 & $3.09_{-0.10}^{+0.10}$ & $1.06_{-0.01}^{+0.01}$ & $0.65_{-0.01}^{+0.01}$ & $2.79_{-0.11}^{+0.11}$ & $0.62_{-0.01}^{+0.01}$ & $0.38_{-0.01}^{+0.01}$ \\
\hline RXC J0145-5300 & 0.1168 & $5.53_{-0.13}^{+0.13}$ & $2.26_{-0.02}^{+0.02}$ & $1.40_{-0.01}^{+0.01}$ & $5.51_{-0.16}^{+0.16}$ & $1.76_{-0.02}^{+0.02}$ & $1.09_{-0.01}^{+0.01}$ \\
\hline RXC J0211-4017 & 0.1008 & $2.07_{-0.00}^{+0.07}$ & $0.55_{-0.00}^{+0.00}$ & $0.34_{-0.00}^{+0.00}$ & $2.02_{-0.06}^{+0.06}$ & $0.33_{-0.00}^{+0.00}$ & $0.20_{-0.00}^{+0.00}$ \\
\hline RXC J0225-2928 & 0.0604 & $2.47_{-0.06}^{+0.15}$ & $0.33_{-0.01}^{+0.01}$ & $0.21_{-0.00}^{+0.00}$ & $2.61_{-0.16}^{+0.16}$ & $0.19_{-0.00}^{+0.00}$ & $0.12_{-0.00}^{+0.00}$ \\
\hline RXC J0345-4112 & 0.0603 & $2.19_{-0.04}^{+0.04}$ & $0.51_{-0.01}^{+0.01}$ & $0.32_{-0.00}^{+0.00}$ & $2.15_{-0.08}^{+0.08}$ & $0.25_{-0.00}^{+0.00}$ & $0.15_{-0.00}^{+0.00}$ \\
\hline RXC J0547-3152 & 0.1483 & $6.02_{-0.11}^{+0.11}$ & $3.88_{-0.02}^{+0.02}$ & $2.40_{-0.01}^{+0.01}$ & $5.68_{-0.11}^{+0.11}$ & $2.58_{-0.02}^{+0.02}$ & $1.59_{-0.01}^{+0.01}$ \\
\hline RXC J0605-3518 & 0.1392 & $4.56_{-0.05}^{+0.05}$ & $4.72_{-0.02}^{+0.02}$ & $2.94_{-0.01}^{+0.01}$ & $4.81_{-0.12}^{+0.12}$ & $2.07_{-0.02}^{+0.02}$ & $1.28_{-0.01}^{+0.01}$ \\
\hline RXC J0616-4748 & 0.1164 & $4.22_{-0.10}^{+0.10}$ & $1.24_{-0.01}^{+0.01}$ & $0.76_{-0.01}^{+0.01}$ & $4.16_{-0.12}^{+0.12}$ & $0.98_{-0.01}^{+0.01}$ & $0.60_{-0.01}^{+0.01}$ \\
\hline RXC J0645-5413 & 0.1644 & $6.95_{-0.13}^{+0.13}$ & $7.57_{-0.04}^{+0.04}$ & $4.69_{-0.03}^{+0.03}$ & $6.97_{-0.19}^{+0.19}$ & $4.57_{-0.03}^{+0.03}$ & $2.83_{-0.02}^{+0.02}$ \\
\hline RXC J0821+0112 & 0.0822 & $2.68_{-0.09}^{+0.09}$ & $0.49_{-0.01}^{+0.01}$ & $0.30_{-0.00}^{+0.00}$ & $2.44_{-0.12}^{+0.12}$ & $0.36_{-0.01}^{+0.01}$ & $0.22_{-0.00}^{+0.00}$ \\
\hline RXC J0958-1103 & 0.1669 & $5.34_{-0.21}^{+0.21}$ & $5.30_{-0.07}^{+0.07}$ & $3.28_{-0.04}^{+0.04}$ & $5.85_{-0.40}^{+0.45}$ & $2.31_{-0.06}^{+0.06}$ & $1.43_{-0.04}^{+0.04}$ \\
\hline RXC J1044-0704 & 0.1342 & $3.41_{-0.03}^{+0.03}$ & $4.24_{-0.01}^{+0.01}$ & $2.62_{-0.01}^{+0.01}$ & $3.52_{-0.05}^{+0.05}$ & $1.70_{-0.01}^{+0.01}$ & $1.04_{-0.01}^{+0.01}$ \\
\hline RXC J1141-1216 & 0.1195 & $3.31_{-0.03}^{+0.03}$ & $2.14_{-0.01}^{+0.01}$ & $1.33_{-0.00}^{+0.00}$ & $3.40_{-0.06}^{+0.06}$ & $0.97_{-0.01}^{+0.01}$ & $0.60_{-0.00}^{+0.00}$ \\
\hline RXC J1236-3354 & 0.0796 & $2.70_{-0.05}^{+0.05}$ & $0.64_{-0.01}^{+0.01}$ & $0.40_{-0.00}^{+0.00}$ & $2.57_{-0.03}^{+0.11}$ & $0.39_{-0.00}^{+0.00}$ & $0.24_{-0.00}^{+0.00}$ \\
\hline RXC J1302-0230 & 0.0847 & $2.97_{-0.07}^{+0.06}$ & $0.83_{-0.01}^{+0.01}$ & $0.51_{-0.00}^{+0.00}$ & $2.92_{-0.07}^{+0.09}$ & $0.51_{-0.00}^{+0.00}$ & $0.31_{-0.00}^{+0.00}$ \\
\hline RXC J1311-0120 & 0.1832 & $8.91_{-0.08}^{+0.08}$ & $12.48_{-0.03}^{+0.03}$ & $7.76_{-0.02}^{+0.00}$ & $8.24_{-0.13}^{+0.01}$ & $5.49_{-0.02}^{+0.02}$ & $3.41_{-0.01}^{+0.01}$ \\
\hline RXC J1516+0005 & 0.1181 & $4.51_{-0.06}^{+0.06}$ & $2.08_{-0.01}^{+0.01}$ & $1.28_{-0.01}^{+0.01}$ & $4.18_{-0.08}^{+0.08}$ & $1.45_{-0.01}^{+0.01}$ & $0.89_{-0.01}^{+0.01}$ \\
\hline RXC J1516-0056 & 0.1198 & $3.55_{-0.07}^{+0.07}$ & $1.30_{-0.01}^{+0.01}$ & $0.80_{-0.01}^{+0.01}$ & $3.40_{-0.08}^{+0.08}$ & $1.02_{-0.01}^{+0.01}$ & $0.62_{-0.01}^{+0.01}$ \\
\hline RXC J2014-2430 & 0.1538 & $4.78_{-0.05}^{+0.05}$ & $10.24_{-0.03}^{+0.03}$ & $6.34_{-0.02}^{+0.01}$ & $5.63_{-0.11}^{+0.08}$ & $3.38_{-0.03}^{+0.01}$ & $2.09_{-0.02}^{+0.01}$ \\
\hline RXC J2023-2056 & 0.0564 & $2.71_{-0.09}^{+0.09}$ & $0.39_{-0.01}^{+0.01}$ & $0.24_{-0.00}^{+0.00}$ & $2.46_{-0.12}^{+0.12}$ & $0.26_{-0.01}^{+0.01}$ & $0.16_{-0.00}^{+0.00}$ \\
\hline RXC J2048-1750 & 0.1475 & $4.65_{-0.07}^{+0.13}$ & $2.55_{-0.01}^{+0.01}$ & $1.57_{-0.01}^{+0.01}$ & $4.59_{-0.08}^{+0.08}$ & $2.21_{-0.01}^{+0.01}$ & $1.36_{-0.01}^{+0.01}$ \\
\hline RXC J2129-5048 & 0.0796 & $3.81_{-0.15}^{+0.01}$ & $0.79_{-0.01}^{+0.01}$ & $0.49_{-0.01}^{+0.01}$ & $3.64_{-0.12}^{+0.08}$ & $0.65_{-0.01}^{+0.01}$ & $0.41_{-0.01}^{+0.01}$ \\
\hline RXC J2149-3041 & 0.1184 & $3.26_{-0.04}^{+0.04}$ & $2.06_{-0.01}^{+0.01}$ & $1.27_{-0.01}^{+0.01}$ & $3.40_{-0.08}^{+0.08}$ & $0.91_{-0.01}^{+0.01}$ & $0.56_{-0.00}^{+0.00}$ \\
\hline RXC J2157-0747 & 0.0579 & $2.46_{-0.08}^{+0.08}$ & $0.29_{-0.00}^{+0.00}$ & $0.18_{-0.00}^{+0.00}$ & $2.30_{-0.06}^{+0.10}$ & $0.25_{-0.00}^{+0.00}$ & $0.15_{-0.00}^{+0.00}$ \\
\hline RXC J2217-3543 & 0.1486 & $4.86_{-0.09}^{+0.09}$ & $2.98_{-0.01}^{+0.01}$ & $1.84_{-0.01}^{+0.01}$ & $4.45_{-0.09}^{+0.09}$ & $1.89_{-0.01}^{+0.01}$ & $1.16_{-0.01}^{+0.01}$ \\
\hline RXC J2218-3853 & 0.1411 & $5.84_{-0.11}^{+0.11}$ & $4.13_{-0.03}^{+0.01}$ & $2.56_{-0.02}^{+0.01}$ & $5.88_{-0.15}^{+0.09}$ & $2.44_{-0.03}^{+0.01}$ & $1.51_{-0.02}^{+0.01}$ \\
\hline RXC J2234-3744 & 0.1510 & $7.78_{-0.15}^{+0.15}$ & $7.20_{-0.04}^{+0.04}$ & $4.47_{-0.03}^{+0.03}$ & $6.95_{-0.14}^{+0.14}$ & $4.96_{-0.04}^{+0.04}$ & $3.07_{-0.02}^{+0.02}$ \\
\hline RXC J2319-7313 & 0.0984 & $2.22_{-0.03}^{+0.03}$ & $1.34_{-0.01}^{+0.01}$ & $0.82_{-0.01}^{+0.01}$ & $2.48_{-0.08}^{+0.08}$ & $0.63_{-0.01}^{+0.01}$ & $0.38_{-0.01}^{+0.01}$ \\
\hline
\end{tabular}

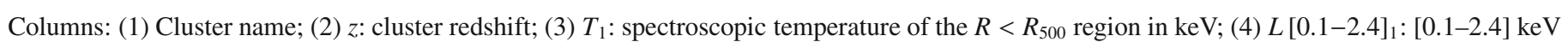
band luminosity in the $R<R_{500}$ region in units of $10^{44} \mathrm{erg} \mathrm{s}^{-1}$; (5) $L[0.5-2]_{1}$ : [0.5-2] keV band luminosity in the $R<R_{500}$ region in units of $10^{44} \mathrm{erg} \mathrm{s}^{-1}$; (6) $T_{2}$ : spectroscopic temperature in the [0.15-1] $R_{500}$ region in keV; (7) $L[0.1-2.4]_{2}:[0.1-2.4] \mathrm{keV}$ band luminosity in the $[0.15-1] R_{500}$ region in units of $10^{44} \mathrm{erg} \mathrm{s}^{-1} ;(8) L[0.5-2]_{2}:[0.5-2] \mathrm{keV}$ band luminosity in the $[0.15-1] R_{500}$ region in units of $10^{44} \mathrm{erg} \mathrm{s}^{-1}$.

The right hand panel of Fig. B.2 shows the corrected [0.5-2] $\mathrm{keV}$ band relation compared to the results derived by Vikhlinin et al. (2008) using the same Malmquist bias correction procedure on a sample of clusters observed with Chandra (the Chandra Cluster Cosmology Project, CCCP). The agreement in normalisation is good at low $L / M$, but at higher $L / M$ the Vikhlinin et al. relation is somewhat below ours (by approximately 40 per cent at $8 \mathrm{keV}$, or $8 \times 10^{14} M_{\odot}$ ).

The REXCESS and CCCP slopes are slightly different, although it is important to note that they are in agreement within their $1 \sigma$ uncertainties. The bias correction itself does not play a part because there is excellent agreement in the magnitude of the scatter about the $L-M$ relation from the two samples. We use a different $M_{500}-Y_{\mathrm{X}}$ relation to estimate masses, although in practice the effect of this difference will be small since our relation is in good agreement with theirs. One partial explanation could be due to the systematic offset in measurements between Chandra and XMM-Newton, in which, at high temperatures, Chandra overestimates the temperature ${ }^{4}$. Since in both cases masses are derived from $Y_{\mathrm{X}}=M_{\mathrm{gas}} T$, this will have the effect of boosting the higher mass Chandra points at a given luminosity, leading to a flatter relation than the one we find here. The effect is of order 20 per cent at $8 \mathrm{keV}$ for a mass calculated from the $M-Y_{\mathrm{X}}$ relation, which alleviates the difference somewhat. Finally, the samples contain different clusters. The individual samples probe slightly different mass ranges as REXCESS contains more lower mass systems, while the local CCCP sample contains more higher mass systems. In addition, differences in the number of cool core systems, and their distribution across the mass range, could change the slope. In particular, if REXCESS

${ }^{4}$ A Comparison of Cluster Temperatures Derived from Chandra and XMM-Newton http://cxc.harvard.edu/cal/memos/ hrma_memo.pdf 
Table B.2. Observed survey band X-ray luminosity scaling relations for the full REXCESS sample. Each set of observables $(L, A)$ is fitted with a power law relation of the form $h(z)^{n} L=C\left(A / A_{0}\right)^{\alpha}$, with $A_{0}=5 \mathrm{keV}$ and $2 \times 10^{14} M_{\odot} \mathrm{keV}$, and $n=-1,-9 / 5$ and $-7 / 3$ for $T, Y_{\mathrm{X}}$ and $M$, respectively. Results are given for the BCES (Y|X) and BCES orthogonal fitting methods (see Sect. 2.4). The intrinsic natural logarithmic scatter about the best fitting relation in the $\ln -\ln$ plane is given in each case.

\begin{tabular}{|c|c|c|c|c|c|c|}
\hline \multirow[t]{3}{*}{$\overline{\overline{\text { Relation }}}$} & \multicolumn{6}{|c|}{ Fitting method } \\
\hline & \multicolumn{3}{|c|}{$\operatorname{BCES}(\mathrm{Y} \mid \mathrm{X})$} & \multicolumn{3}{|c|}{ BCES orthogonal } \\
\hline & $C\left(10^{44} \mathrm{erg} \mathrm{s}^{-1}\right)$ & $\alpha$ & $\sigma_{\ln \mathrm{L}, \text { intrinsic }}$ & $C\left(10^{44} \mathrm{erg} \mathrm{s}^{-1}\right)$ & $\alpha$ & $\sigma_{\ln \mathrm{L}, \text { intrinsic }}$ \\
\hline & \multicolumn{6}{|c|}{$R<R_{500}$} \\
\hline$L[0.1-2.4]_{1}-T_{1}$ & $2.86 \pm 0.27$ & $2.24 \pm 0.22$ & $0.665 \pm 0.119$ & $3.46 \pm 0.55$ & $3.00 \pm 0.35$ & $0.757 \pm 0.144$ \\
\hline$L[0.5-2]_{1}-T_{1}$ & $1.77 \pm 0.17$ & $2.24 \pm 0.22$ & $0.666 \pm 0.119$ & $2.14 \pm 0.34$ & $3.01 \pm 0.35$ & $0.758 \pm 0.144$ \\
\hline$L[0.1-2.4]_{1}-Y_{\mathrm{X}}$ & $2.52 \pm 0.18$ & $0.84 \pm 0.05$ & $0.411 \pm 0.070$ & $2.60 \pm 0.20$ & $0.90 \pm 0.06$ & $0.412 \pm 0.071$ \\
\hline$L[0.5-2]_{1}-Y_{\mathrm{X}}$ & $1.56 \pm 0.11$ & $0.84 \pm 0.06$ & $0.413 \pm 0.07$ & $1.61 \pm 0.12$ & $0.90 \pm 0.06$ & $0.414 \pm 0.071$ \\
\hline$L[0.1-2.4]_{1}-M_{Y}$ & $1.03 \pm 0.08$ & $1.53 \pm 0.10$ & ${ }^{a} \ldots$ & $0.98 \pm 0.07$ & $1.71 \pm 0.12$ & ${ }^{a} \ldots$ \\
\hline$L[0.1-2.4]_{1}-M_{Y} \mathrm{MB}^{b}$ & $0.83 \pm 0.07$ & $1.62 \pm 0.11$ & ${ }^{a} \ldots$ & $0.78 \pm 0.07$ & $1.83 \pm 0.14$ & $a_{\ldots} \ldots$ \\
\hline$L[0.5-2]_{1}-M_{Y}$ & $0.64 \pm 0.05$ & $1.53 \pm 0.10$ & ${ }^{a} \ldots$ & $0.61 \pm 0.05$ & $1.72 \pm 0.12$ & ${ }^{a} \ldots$ \\
\hline$L[0.5-2]_{1}-M_{Y} \mathrm{MB}^{b}$ & $0.51 \pm 0.04$ & $1.62 \pm 0.12$ & ${ }^{a} \ldots$ & $0.48 \pm 0.04$ & $1.83 \pm 0.14$ & ${ }^{a} \ldots$ \\
\hline
\end{tabular}

\begin{tabular}{rllllll}
$L[0.1-2.4]_{2}-T_{2}$ & $1.85 \pm 0.09$ & $2.32 \pm 0.13$ & $0.278 \pm 0.056$ & $1.95 \pm 0.12$ & $2.52 \pm 0.16$ & $0.293 \pm 0.062$ \\
$L[0.5-2]_{2}-T_{2}$ & $1.14 \pm 0.05$ & $2.34 \pm 0.13$ & $0.276 \pm 0.056$ & $1.20 \pm 0.07$ & $2.53 \pm 0.16$ & $0.291 \pm 0.062$ \\
& & & & & & \\
$L[0.1-2.4]_{2}-Y_{\mathrm{X}}$ & $1.50 \pm 0.04$ & $0.82 \pm 0.03$ & $0.174 \pm 0.044$ & $1.51 \pm 0.04$ & $0.83 \pm 0.03$ & $0.175 \pm 0.044$ \\
$L[0.5-2]_{2}-Y_{\mathrm{X}}$ & $0.92 \pm 0.02$ & $0.82 \pm 0.03$ & $0.173 \pm 0.044$ & $0.93 \pm 0.03$ & $0.83 \pm 0.03$ & $0.174 \pm 0.044$ \\
& & & & & \\
$L[0.1-2.4]_{1}-M_{Y}$ & $0.63 \pm 0.03$ & $1.49 \pm 0.05$ & ${ }^{a} \ldots$ & $0.62 \pm 0.03$ & $1.52 \pm 0.05$ & ${ }^{a} \ldots$ \\
$L[0.5-2]_{1}-M_{Y}$ & $0.39 \pm 0.02$ & $1.49 \pm 0.05$ & $a_{\ldots}$ & $0.38 \pm 0.02$ & $1.53 \pm 0.05$ & $a^{\ldots}$ \\
\hline
\end{tabular}

$L_{1} / T_{1}$ : luminosity/temperature interior to $R_{500} ; L_{2} / T_{2}$ : luminosity/temperature in the $[0.15-1] R_{500}$ aperture; $M_{Y}$ : total mass estimated from the $M_{500}-Y_{\mathrm{X}}$ relation of Arnaud et al. (2007). ${ }^{a}$ Since $M$ is derived from $Y_{\mathrm{X}}$, the values of the scatter in the $L-M$ relation are identical to those for the $L-Y_{\mathrm{X}}$ relation; ${ }^{b}$ relations corrected for Malmquist bias.
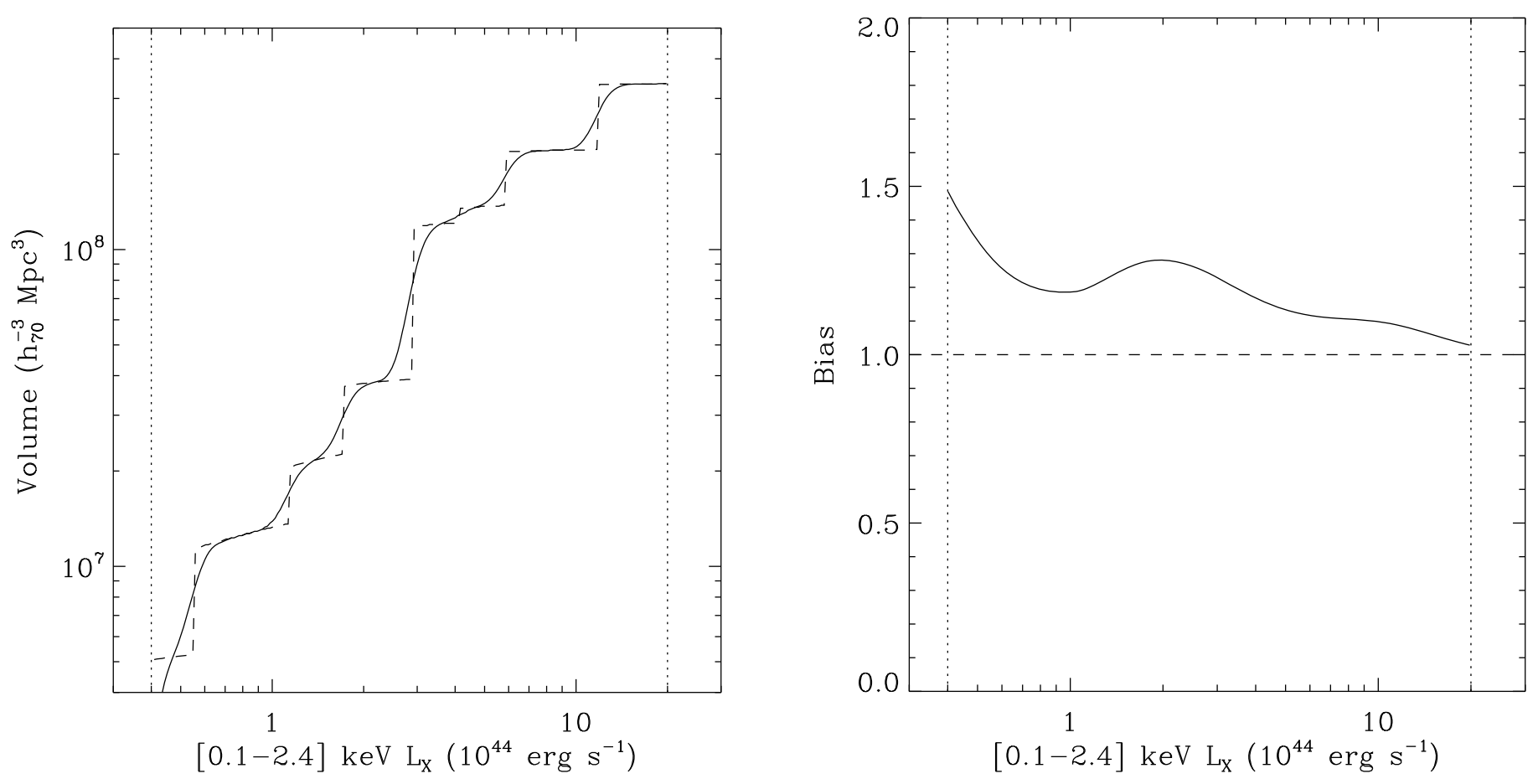

Fig. B.1. Left panel: survey volume for the REXCESS sample. The dashed line is the raw survey volume; the solid line is the volume folded with an assumed measurement error of 10 per cent on the luminosity. Right panel: malmquist bias for the REXCESS sample as a function of luminosity. 

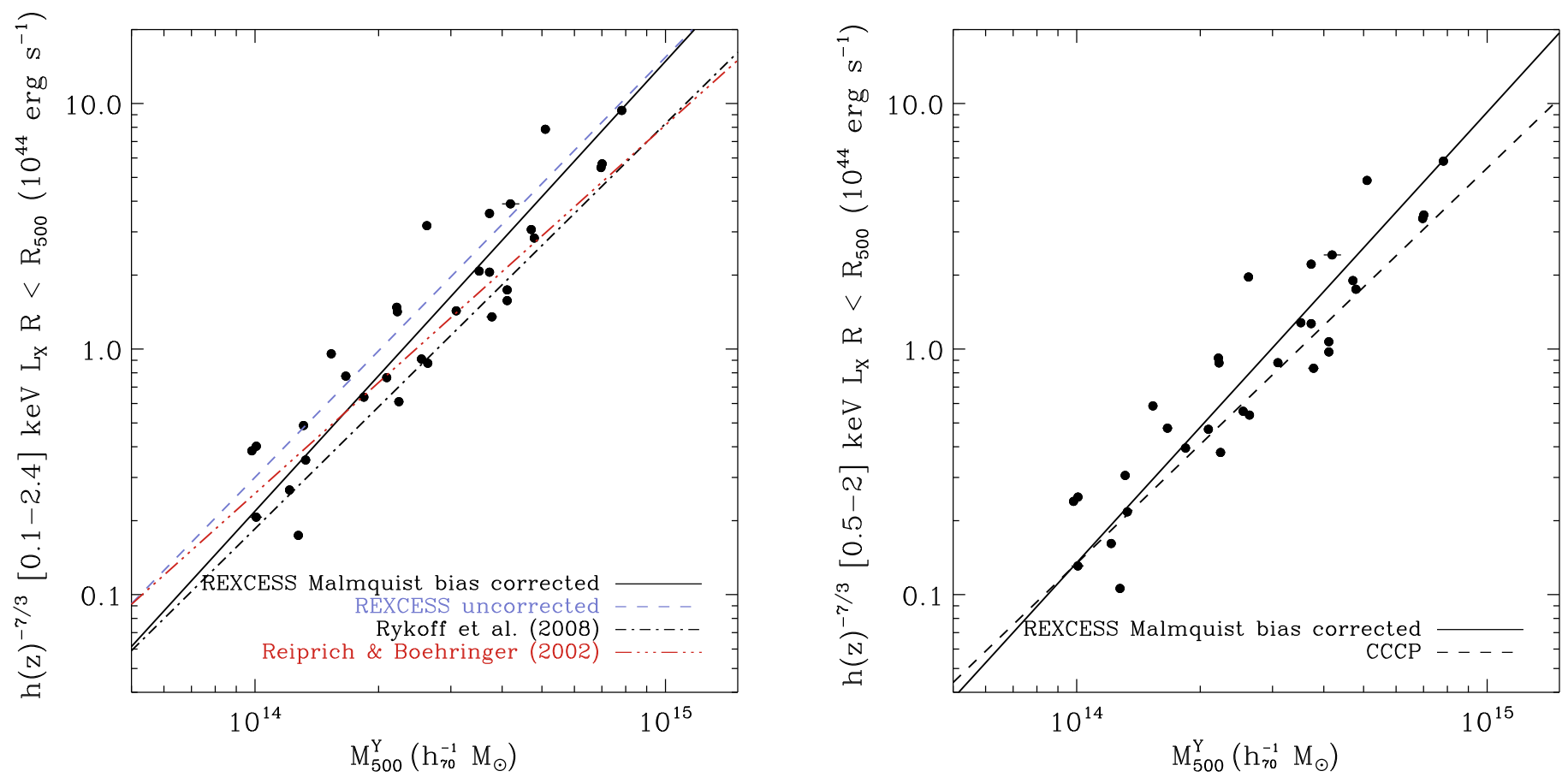

Fig. B.2. Left panel: the $L[0.1-2.4]-M_{Y}$ relation compared with previous determinations from X-ray hydrostatic analysis assuming isothermality (Reiprich \& Böhringer 2002) and stacked weak lensing analysis (Rykoff et al. 2008). The points are the bias-corrected REXCESS values. Right panel: malmquist bias corrected $L[0.5-2]-M_{\mathrm{Y}}$ relation compared with the results from Vikhlinin et al. (2008). The best fitting relation is given in Table B.2. All fits have been undertaken with the BCES orthogonal fitting method.

has more cool core systems at higher mass than CCCP, their higher luminosity would make the REXCESS relation slightly steeper.

\section{References}

Akritas, M. G., \& Bershady, M. A. 1996, ApJ, 470, 706

Allen, S. W., \& Fabian, A. C. 1998, MNRAS, 297, L57

Arnaud, M., \& Evrard, A. E. 1999, MNRAS, 305, 631

Arnaud, M., Pointecouteau, E., \& Pratt, G. W. 2005, A\&A, 441, 893

Arnaud, M., Pointecouteau, E., \& Pratt, G. W. 2007, A\&A, 474, L37

Böhringer, H., Schuecker, P., Guzzo, L., et al. 2001, A\&A, 369, 826

Böhringer, H., Schuecker, P., Guzzo, L., et al. 2004, A\&A, 425, 367

Böhringer, H., Schuecker, P., Pratt, G. W., et al. 2007, A\&A, 469, 363

Bower, R. G., McCarthy, I. G., \& Benson, A. J. 2008, MNRAS, 390, 1399

Croston, J. H., Arnaud, M., Pointecouteau, E., \& Pratt, G. W. 2006, A\&A, 459, 1007

Croston, J. H., Pratt, G. W., Böhringer, H., et al. 2008, A\&A, 487, 431

David, L. P., Slyz, A., Jones, C., et al. 1993, ApJ, 412, 479

Dunkley, J., Komatsu, E., Nolta, M. R., et al. 2009, ApJS, 180, 306

Edge, A. C., \& Stewart, G. C. 1991, MNRAS, 252, 414

Fabian, A. C., Crawford, C. S., Edge, A. C., \& Mushotzky, R. F. 1994, MNRAS, 267, 779

Gastaldello, F., Buote, D. A., Humphrey, P. J., et al. 2007, ApJ, 669, 158

Hartley, W. G., Gazzola, L., Pearce, F. R., Kay, S. T., \& Thomas, P. A. 2008 , MNRAS, 386, 2015

Henry, P., \& Tucker, W. 1979, ApJ, 229, 78

Ikebe, Y., Reiprich, T. H., Böhringer, H., Tanaka, Y., \& Kitayama, T. 2002, A\&A, 383, 773

Isobe, T., Feigelson, E. D., Akritas, M. G., \& Babu, G. J. 1990, ApJ, 364, 104

Kravtsov, A. V., Vikhlinin, A., \& Nagai, D. 2006, ApJ, 650, 128

Lima, M., \& Hu, W. 2005, Phys. Rev. D, 72, 043006
Markevitch, M. 1998, ApJ, 504, 27

Maughan, B. J. 2007, ApJ, 668, 772

Mitchell, R. J., Ives, J. C., \& Culhane, J. L. 1977, MNRAS, 181, 25P

Mohr, J. J., Fabricant, D. G., \& Geller, M. J. 1993, ApJ, 413, 492

Mohr, J. J., Mathiesen, B., \& Evrard, A. E. 1999, ApJ, 517, 627

Mushotzky, R. F., Serlemitsos, P. J., Boldt, E. A., Holt, S. S., \& Smith, B. W. 1978, ApJ, 225, 21

Nagai, D., Kravtsov, A. V., \& Vikhlinin, A. 2007, ApJ, 668, 1

Neumann, D. M., \& Arnaud, M. 1999, A\&A, 348, 711

Neumann, D. M., \& Arnaud, M. 2001, A\&A, 373, L33

Novicki, M. C., Sornig, M., \& Henry, J. P. 2002, AJ, 124, 2413

O'Hara, T. B., Mohr, J. J., Bialek, J. J., \& Evrard, A. E. 2006, ApJ, 639, 64

Osmond, J. P. F., \& Ponman, T. J. 2004, MNRAS, 350, 1511

Pacaud, F., Pierre, M., Adami, C., et al. 2007, MNRAS, 382, 1289

Pointecouteau, E., Arnaud, M., \& Pratt, G. W. 2005, A\&A, 435, 1

Poole, G. B., Babul, A., McCarthy, I. G., et al. 2007, MNRAS, 380, 437

Pratt, G. W., Arnaud, M., \& Pointecouteau, E. 2006, A\&A, 446, 429

Pratt, G. W., Böhringer, H., Croston, J. H., et al. 2007, A\&A, 461, 71

Puchwein, E., Sijacki, D., \& Springel, V. 2008, ApJ, 687, L53

Reiprich, T. H., \& Böhringer, H. 2002, ApJ, 567, 716

Ricker, P. M., \& Sarazin, C. L. 2001, ApJ, 561, 621

Ritchie, B. W., \& Thomas, P. A. 2002, MNRAS, 329, 675

Rykoff, E. S., Evrard, A. E., McKay, T. A., et al. 2008, MNRAS, 387, L28

Stanek, R., Evrard, A. E., Böhringer, H., Schuecker, P., \& Nord, B. 2006, ApJ, 648,956

Sun, M., Voit, G. M., Donahue, M., Jones, C., \& Forman, W. 2008, ApJ, in press [arXiv:0805.2320]

Vikhlinin, A., Kravtsov, A., Forman, W., et al. 2006, ApJ, 640, 691

Vikhlinin, A., Burenin, R. A., Ebeling, H., et al. 2008, ApJ, submitted [arXiv:0805.2207]

Yang, H. Y., Ricker, P. M., \& Sutter, P. M. 2008, ApJ, submitted [arXiv: 0808.4099] 